\title{
Iwasawa's Local Splitting Theorem for Pro-Lie Groups
}

\author{
Karl H. Hofmann and Sidney A. Morris \\ (Communicated by Karl-Hermann Neeb)
}

\begin{abstract}
If the nilradical $\mathfrak{n}(\mathfrak{g})$ of the Lie algebra $\mathfrak{g}$ of a pro-Lie group $G$ is finite dimensional modulo the center $z(\mathfrak{g})$, then every identity neighborhood $U$ of $G$ contains a closed normal subgroup $N$ such that $G / N$ is a Lie group and $G$ and $N \times G / N$ are locally isomorphic.
\end{abstract}

2000 Mathematics Subject Classification: 22E65, 17B65, 22A05; 22E15, 22E60, 22E25, 22D05.

\section{Background and Introduction}

A local morphism from a topological group $G$ to a topological group $H$ is a continuous function $\lambda: U \rightarrow V, V \subseteq H$, defined on an open identity neighborhood $U$ of $G$ such that $g, g^{\prime}, g g^{\prime} \in U$ implies $\lambda\left(g g^{\prime}\right)=\lambda(g) \lambda\left(g^{\prime}\right)$. A local morphism $\lambda$ is called a local isomorphism if it has an inverse $\lambda^{-1}: V \rightarrow U$ which is a local morphism from $H$ to $G$. If a local isomorphism from $G$ to $H$ exists, then $G$ and $H$ are said to be locally isomorphic.

For a closed normal subgroup $N$ of $G$ let $q: G \rightarrow G / N$ be the quotient morphism. Then $N$ is said to split locally if there is a local morphism $\lambda: U \rightarrow V$ from $G / N$ to $G$ such that for the inclusion map $j: V \rightarrow G$, the composition $q \circ j \circ \lambda$ is the identity map of $U$, that is, that $q(\lambda(u))=u$ for all $u \in U \subseteq G / N$.

Assume that $N$ is a normal co-Lie subgroup, that is, $G / N$ is a Lie group. Then let $L$ be the simply connected covering group of the identity component $(G / N)_{0}$ and let $p: L \rightarrow G / N$ be the coextension of the covering morphism. Let $W$ be a connected symmetric open identity neighborhood of $L$ such that $p(W) \subseteq U$. Then by the simple connectivity of $L$ there is a unique morphism $f: L \rightarrow G$ such that $f(x)=\lambda(p(x))$ for all $x \in W$ (see for instance [2], Corollary A2.26). There is a morphism $\alpha: L \rightarrow \operatorname{Aut}(N)$ given by $\alpha(x)(n)=f(x) n f(x)^{-1}$, allowing us to form the semidirect product $N \rtimes_{\alpha} L$ and the morphism

$$
\mu: N \rtimes L \rightarrow G, \quad \mu(n, x)=n f(x)
$$

whose kernel is readily seen to be $\left\{\left(f(x)^{-1}, x\right): x \in f^{-1}(N)\right\} \cong f^{-1}(N)$, a discrete subgroup of $N \rtimes_{\alpha} L$ and whose image is the open subgroup of $G$ generated by $N \lambda(U)$. 
This simple and straightforward process allows us to view a closed normal co-Lie subgroup $N$ of $G$ that splits locally as being given by an open morphism with discrete kernel from a semidirect product $N \rtimes L$ of $N$ with a Lie group $L$. In particular, $N \rtimes L$ and $G$ are locally isomorphic, as are $L$ and $G / N$.

A closed normal subgroup of $N$ is said to split locally and directly if the image of some defining local morphism $\lambda: U \rightarrow V$ from $G / N \rightarrow G$ commutes elementwise with $N$. Then, if $G / N$ is a Lie group, this happens if the semidirect product $N \rtimes_{\alpha} L$ is direct and thus $G$ and $N \times G / N$ are locally isomorphic. Iwasawa's influential paper [10] of 1949 contains the following powerful theorem:

Iwasawa Local Splitting Theorem. Let $G$ be a connected locally compact group and $U$ an identity neighborhood of $G$. Then there is a closed normal co-Lie subgroup $N$ contained in $U$ that splits locally and directly. In particular, $G$ and $N \times G / N$ are locally isomorphic.

Historically, our rendition of Iwasawa's is not quite accurate. He formulated this theorem for connected locally compact groups which are projective limits of Lie groups. It was proved only three or four years later by Yamabe $[13,14]$ that every connected locally compact group is indeed a projective limit of Lie groups.

Definition. A topological group is a pro-Lie group if it is complete and every identity neighborhood contains a normal co-Lie subgroup.

Thus every connected locally compact group is a particular example of a pro-Lie group. Better still: A topological group $G$ is said to be almost connected if the quotient group $G / G_{0}$ modulo its component is compact. Yamabe's Theorem even establishes that any almost connected locally compact group is a pro-Lie group.

Evidently, a Local Splitting Theorem such as Iwasawa's is of great power and significance for the structure theory of connected locally compact groups and indeed many authors have used it in one way or another. This motivates us to investigate local splitting for the class of pro-Lie groups in general.

It is shown in [1], [4], and [9] that a topological group is a pro-Lie group if and only if it is the limit of a projective system of finite dimensional Lie groups; it is not trivial that this condition is sufficient. Given this equivalence, it is not hard to recognize another equivalent condition, namely, that $G$ be isomorphic as a topological group to a closed subgroup of a product of finite dimensional Lie groups (see [9]).

This paper is concerned with the local direct splitting of arbitrarily small closed normal co-Lie subgroups of pro-Lie groups. Unfortunately, the local splitting of small normal co-Lie subgroups does not hold for arbitrary pro-Lie groups, as we shall see by exhibiting examples. So our challenge is to find and describe the general obstruction to local splitting.

Our procedures require some prerequisites, notably, on the Lie theory of pro-Lie groups for which we refer to recent papers [4] and [6]; a comprehensive source will become available shortly [9]. The first step is to investigate the local splitting of normal co-Lie subgroups of pro-Lie groups in the spirit of our introductory remarks. 
The second step will be the study of the local splitting on the Lie algebra level, while the third and final step is the local splitting on the group level.

Acknowledgment. The authors thank the referee for a careful scrutiny of their text which helped them to avoid a number of typographical errors and led to various revisions that improved the presentation.

\section{Locally Splitting Lie Group Quotients of Pro-Lie Groups}

For our dealing with pro-Lie groups, we have pursued the strategy of preparing a structure theoretical idea by first working as much as we can on the level of the proLie algebras. We begin by proving a result that will motivate and justify why we should focus on the pro-Lie algebra level.

Let $G$ be a connected pro-Lie group with Lie algebra $\mathfrak{g}=\mathfrak{L}(G)$ and $N$ a closed normal subgroup with Lie algebra $\mathfrak{n}=\mathfrak{L}(N)$. Assume that $\mathfrak{g}$ is the semidirect sum $\mathfrak{n} \oplus \mathfrak{h}$ of the closed ideal $\mathfrak{n}$ and a closed subalgebra $\mathfrak{h}$. We recall from [6] or [9], Theorem 6.6(vi), that the functors $\Gamma:$ proILIE $\mathbb{A} \mathbb{L} \mathbb{G} \rightarrow$ simpconnproILIIEG $\mathbb{R}$ and $\mathfrak{L}:$ simpconnproILIIEG $\mathbb{R} \rightarrow$ proILIIE $\mathbb{A} \mathbb{L} \mathbb{G}$ implement an equivalence of categories between the category of pro-Lie algebras and that of simply connected pro-Lie groups. Define $H \stackrel{\text { def }}{=} \Gamma(\mathfrak{h})$, the unique simply connected pro-Lie group with Lie algebra $\mathfrak{h}$ according to [6] and [9]. The inclusion $i_{\mathfrak{h}}: \mathfrak{h} \rightarrow \mathfrak{g}$ induces a morphism $\Gamma\left(i_{\mathfrak{h}}\right): H \rightarrow \tilde{G} \stackrel{\text { def }}{=} \Gamma(\mathfrak{g})$. Recall that we have a natural morphism $\pi_{G}: \tilde{G} \rightarrow G$, called the universal morphism. (It is obtained as the back adjunction of the pair of adjoint functors $(\Gamma, \mathfrak{L})$ and reduces, in the case of connected Lie groups, to the universal covering morphism.) We set $\varphi \stackrel{\text { def }}{=} \pi_{G} \circ \Gamma\left(i_{\mathfrak{h}}\right): H \rightarrow G$. Then $\varphi$ is a morphism of topological groups. Now we can define a group homomorphism $\alpha: H \rightarrow \operatorname{Aut}(N)$ by $\alpha(h)(n)=\varphi(h) n \varphi(h)^{-1}$ and see immediately that the automorphic action

$$
(h, n) \mapsto \alpha(h)(n): H \times N \rightarrow N
$$

is continuous (irrespective of a potential topology on $\operatorname{Aut}(N)$ ). Therefore the semidirect product $N \rtimes_{\alpha} H$, having the multiplication

$$
\left(n_{1}, h_{1}\right)\left(n_{2}, h_{2}\right)=\left(n_{1} \alpha\left(h_{1}\right)\left(n_{2}\right), h_{1} h_{2}\right)
$$

is well-defined. The reader should be alerted to the fact that for a subalgebra $\mathfrak{h}$ of a Lie algebra $\mathfrak{g}$, the inclusion $i_{\mathfrak{h}}: \mathfrak{h} \rightarrow \mathfrak{g}$ may not induce an embedding $\Gamma\left(i_{\mathfrak{h}}\right): \Gamma(\mathfrak{h}) \rightarrow \Gamma(\mathfrak{g})$ in general even in the case of Lie groups: If $G=\tilde{G}$ is $\mathbb{S}^{3} \cong \mathrm{SU}(2)$, the unit quaternions under multiplication with $\mathfrak{g}=\mathbb{R} \cdot i+\mathbb{R} \cdot j+\mathbb{R} \cdot k$ the set of pure quaternions as Lie algebra and with the exponential function $\exp z=e^{z}=1+z+\frac{1}{2} z^{2}+\cdots$, and if $\mathfrak{h}=\mathbb{R} \cdot i \subseteq \mathfrak{g}$, then $\Gamma(\mathfrak{h}) \cong \mathbb{R}$ while $\operatorname{im} \Gamma\left(i_{\mathfrak{h}}\right)=\left\langle\exp _{\mathbb{S}^{3}} \mathfrak{h}\right\rangle=e^{\mathbb{R} \cdot i}=\mathbb{S}^{1} \subseteq \mathbb{C}$ is a circle subgroup of $\mathbb{S}^{3}$ and so $\Gamma\left(i_{\mathfrak{h}}\right): \Gamma(\mathfrak{h}) \rightarrow \Gamma(\mathfrak{g})=\tilde{G}$ is not injective.

We shall retain the notation we have just introduced in the following result: 
Proposition 1.1 (The Splitting and Sandwich Theorem). Let $N$ be a closed normal subgroup of a pro-Lie group $G$ and assume that the Lie algebra $\mathfrak{g}$ of $G$ is the semidirect sum of the Lie algebra $\mathrm{n}$ of $N$ and some closed subalgebra $\mathfrak{h}$ of the Lie algebra $\mathfrak{g}$ of $G$. Then

(i) there is a simply connected pro-Lie group $H$ with Lie algebra $\mathfrak{h} \cong \mathfrak{g} / \mathfrak{n}$, a semidirect product $N \rtimes_{\alpha} H$ and two morphisms

$$
\tilde{G} \stackrel{\pi_{N \rtimes_{\alpha} H}}{\longrightarrow} N \rtimes_{\alpha} H \stackrel{\mu}{\longrightarrow} G
$$

whose composition $\mu \circ \pi_{N \rtimes_{\alpha} H}$ is the universal morphism $\pi_{G}: \tilde{G} \rightarrow G$.

(ii) Both kernels $\operatorname{ker} \pi_{N \rtimes_{\alpha} H}$ and $\operatorname{ker} \mu$ are totally disconnected, and all of the morphisms $\pi_{N \rtimes_{\alpha} H}$, $\mu$, and $\pi_{G}$ induce isomorphisms $\mathfrak{Q}\left(\pi_{N \rtimes_{\alpha} H}\right), \mathfrak{L}(\mu)$, and $\mathfrak{Q}\left(\pi_{G}\right)$ on the Lie algebra level.

(iii) The function

$$
h \mapsto\left(\varphi(h)^{-1}, h\right): \varphi^{-1}(N) \rightarrow \operatorname{ker} \mu, \quad \varphi: H \rightarrow G,
$$

is an isomorphism of prodiscrete groups.

(iv) The semidirect product $N \rtimes_{\alpha} H$ is a pro-Lie group.

Proof. (i) Since $\mathfrak{h} \cong \mathfrak{g} / \mathfrak{n}$, by [4], Corollary 6.7(i) or Corollary 4.21(i) of [9], the Lie algebra $\mathfrak{h}$ may be identified with the Lie algebra $\mathfrak{L}(G / N)$. We set $H \stackrel{\text { def }}{=} \Gamma(\mathfrak{h})$ and obtain in this fashion a simply connected pro-Lie group whose Lie algebra $\mathfrak{h}$ agrees with that of $G / N$. By the remarks preceding the theorem we have an automorphic action of $H$ on $N$ via the morphism $\alpha: H \rightarrow \operatorname{Aut}(N)$ so that $N \rtimes_{\alpha} H$ is well-defined. It remains to show that we may identify $\tilde{G}$ with $\left(\tilde{N} \rtimes_{\alpha} H\right)^{\sim}$ in a natural way, to define $\mu$, and to verify $\mu \circ \pi_{N \rtimes_{\alpha} H}=\pi_{G}$.

By the Strict Exactness Theorem 6.7 of [9], we may identify $\tilde{N}=\Gamma(\mathrm{n})$ with a closed normal subgroup of $\tilde{G}=\Gamma(\mathfrak{g})$ in such a fashion that $\pi_{N}=\pi_{G} \mid \tilde{N}$. For any $h \in H$, we shall abbreviate $\Gamma\left(i_{\mathfrak{h}}\right)(h)$ by $\tilde{h}$. Now by Theorem 6.11 of [9] on the Preservation of Semidirect Products, there is a natural isomorphism

$$
m: \tilde{N} \rtimes_{l} H=\Gamma(\mathfrak{n}) \rtimes_{l} \Gamma(\mathfrak{h}) \rightarrow \tilde{G}=\Gamma(\mathfrak{g}),
$$

$m(x, h)=x \Gamma\left(i_{\mathfrak{h}}\right)(h), x \in \tilde{N} \subseteq \tilde{G}, h \in H$, where the action of $H$ on the normal subgroup $\tilde{N}$ of $\tilde{G}$ is by inner automorphisms so that $\imath(h)(x)=\tilde{h} x \tilde{h}^{-1}$. In particular, $\tilde{N} \rtimes_{l} H$ is a pro-Lie group. Let us define $\mu: N \rtimes_{\alpha} H \rightarrow G$ by $\mu(n, h)=n \varphi(h)$. Then we claim to have a commutative diagram of morphisms

$(*)$

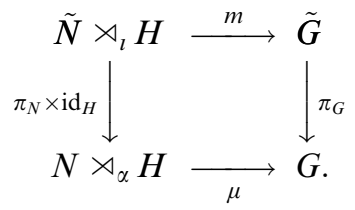


Indeed, for $(x, h) \in \tilde{N} \times H$ we have $\mu \circ\left(\pi_{N} \times \operatorname{id}_{H}\right)(x, h)=\pi_{N}(x) \varphi(h)=\pi_{G}(x) \pi_{G}(\tilde{h})=$ $\pi_{G}(x \tilde{h})=\pi_{G}(m(x, h))$, which establishes our claim. If we identify $\mathfrak{Q}(\tilde{G})$ with $\mathfrak{g}$ so that $\mathfrak{L}\left(\pi_{G}\right)$ becomes the identity and therefore $\mathfrak{L}(\tilde{N})$ becomes identified with $\mathfrak{n}$, applying $\mathfrak{L}$ to the diagram $(*)$ we obtain the commuting diagram

$(* *)$

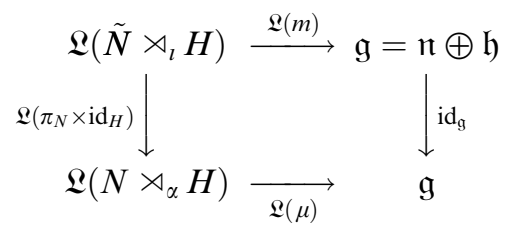

of isomorphisms of pro-Lie algebras. It follows that $\left(\pi_{N} \times \mathrm{id}_{H}\right) \circ m^{-1}: \tilde{G}=\Gamma(\mathfrak{g}) \rightarrow$ $N \rtimes_{\alpha} H$ is $\pi_{N \rtimes_{\alpha} H}$. This completes the proof of (i).

(ii) We have just observed that $\mathfrak{L}\left(\pi_{N \rtimes_{\alpha} H}\right), \mathfrak{L}(\mu)$, and $\mathfrak{L}\left(\pi_{G}\right)$ are isomorphisms. This implies that their kernels are zero. But since $\mathfrak{L}$ preserves kernels, it follows from 3.30 or 4.23 of [9] that the kernels of $\pi_{N \rtimes_{\alpha} H}, \mu$, and $\pi_{G}$ are totally disconnected.

(iii) We have $(n, h) \in \operatorname{ker} \mu$ iff $n=\varphi(h)^{-1} \in N \cap \varphi(H)$ iff $h \in \varphi^{-1}(N)$ and $n=\varphi(h)^{-1}$. Thus $\beta: \varphi^{-1}(N) \rightarrow \operatorname{ker} \mu, \beta(h)=\left(\varphi(h)^{-1}, h\right)$ is a bijective morphism having the inverse given by $\beta^{-1}(n, h)=h$ and is therefore an isomorphism between totally disconnected pro-Lie groups and consequently prodiscrete groups.

(iv) Let $M \in \mathscr{N}(G)$ and $K \in \mathscr{N}(H)$, where $\mathscr{N}(G)$ is the set of normal co-Lie subgroups of $G$. Then $(M \cap N) \times K$ is a normal subgroup of $N \rtimes_{\alpha} H$, as is readily verified. (For instance,

$$
(1, h)(m, k)\left(1, h^{-1}\right)=\left(\varphi(h) m \varphi(h)^{-1}, h k h^{-1}\right) \in(M \cap N) \times K
$$

if $m \in M \cap N$ and $k \in K)$. But $\left(N \rtimes_{\alpha} H\right) /(M \times K) \cong N /(N \cap M) \rtimes H / K$ is a semidirect product of Lie groups and is therefore a Lie group.

Recall that semidirect products of pro-Lie groups need not be pro-Lie groups as is exemplified by such locally compact groups as $(\mathbb{Z} / 2 \mathbb{Z})^{\mathbb{Z}} \rtimes \mathbb{Z}$ with $\mathbb{Z}$ acting automorphically on the power $(\mathbb{Z} / 2 \mathbb{Z})^{\mathbb{Z}}$ via the shift operation.

While $G / N$ is not guaranteed to be a pro-Lie group, it does have a Lie algebra isomorphic to $\mathfrak{g} / \mathfrak{h} \cong \mathfrak{h}$, and by the definition in [6], Theorem 4.6(ii") or in [9], Theorem $2.22\left(\mathrm{i}^{\prime \prime}\right)$, we have $G / N=\Gamma(\mathfrak{g} / \mathfrak{n})=\Gamma(\mathfrak{h})=H$.

Let $q: G \rightarrow G / N$ denote the quotient morphism. Then the situation is described in the following commutative diagram:

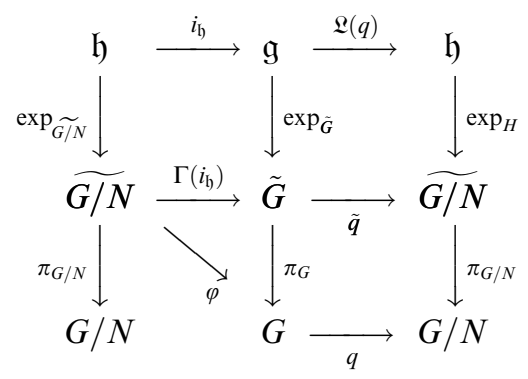


where the top horizontal rows compose to the respective identity morphism and where we recall $\varphi=\pi_{G} \circ \Gamma\left(i_{\mathfrak{h}}\right)$.

We shall assume that $G / N$ is a Lie group shortly after the following corollary resumes the hypotheses of the preceding proposition and imposes additional ones.

Recall that a semidirect sum splitting $\mathfrak{g} \equiv \mathfrak{n} \oplus \mathfrak{h}$ implies that $\mathfrak{h} \cong \mathfrak{g} / \mathfrak{n}$, which allowed us to identify $\Gamma(\mathfrak{h})$ and $\Gamma(\mathfrak{g} / \mathfrak{n})=\widehat{G / N}$ in view of the further natural isomorphism $\mathfrak{g} / \mathfrak{n}=\mathfrak{L}(G) / \mathfrak{L}(N) \cong \mathfrak{L}(G / N)$ of [4], Corollary 6.7(i), or [9], Corollary 4.21(i).

In $[9], 12.88$ (see also $[7,8]$ ) we prove the

Alternative Open Mapping Theorem. Let $f: G \rightarrow H$ be a surjective morphism between pro-Lie groups and assume that

(a) $G / \operatorname{ker} f$ is a pro-Lie group.

(b) $H$ is connected.

(c) $\mathfrak{L}(f): \mathfrak{L}(G) \rightarrow \mathfrak{L}(H)$ is surjective.

Then $f$ is open.

We shall use this in the proof of the following

Corollary 1.2. Assume the hypotheses of Proposition 1.1 and the following hypotheses:

(v) The universal morphism $\pi_{G / N}: \widetilde{G / N} \rightarrow G / N$ is surjective.

(vi) The quotient group $\left(N \rtimes_{\alpha} \widetilde{G / N}\right) / \operatorname{ker} \mu$ is a pro-Lie group.

Then the morphism $\mu: N \rtimes_{\alpha} \widetilde{G / N} \rightarrow G$ defined by $\mu(n, h)=n \varphi(h)$ as in Proposition 1.1 is a quotient morphism with a kernel isomorphic to the central prodiscrete subgroup $\varphi^{-1}(N)$ of $\widehat{G / N}$.

Proof. From the diagram $(\dagger)$ we extract the commutative diagram

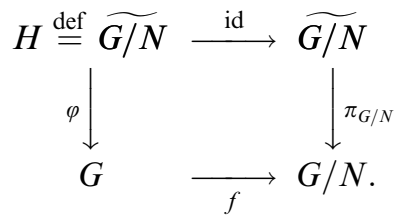

Hypothesis (v) yields the surjectivity of $\pi_{G / N}$, and thus that of $f \circ \varphi$. So let $g \in G$. Then there is an $h \in \widetilde{G / N}$ such that $f(g)=f(\varphi(h))$. Therefore $g \varphi(h)^{-1} \in \operatorname{ker} f=N$, that is there is an $n \in N$ such that $g \varphi(h)^{-1}=n$; so $g=n \varphi(h)=\mu(n, h)$. Hence $\mu: N \rtimes_{\alpha} H \rightarrow G$ is surjective. Now we invoke the Alternative Open Mapping Theorem. Indeed its Hypothesis (a) is satisfied by Assumption (vi) above, Hypothesis (b) is the connectivity of $G / N$, and Hypothesis (iii) is satisfied since $\mathfrak{L}(\mu)$ is an iso- 
morphism by 1.1(ii). Therefore $\mu$ is a quotient morphism and its kernel was identified in 1.1(iii).

The situation is particularly clear if $G / N$ is a Lie group, in which case $\widetilde{G / N}=H$ is the universal covering group of $(G / N)_{0}$. Recall that $\mathscr{N}(G)$ is the filter basis of all normal subgroups $N$ such that $G / N$ is a Lie group. Further recall from Chapter 9 of [9] that for a pro-Lie group $G$ and a closed subalgebra $\mathfrak{h}$ of the Lie algebra $\mathfrak{g}=\mathfrak{L}(G)$ of $G$ we denote by $A(\mathfrak{h})$ the unique smallest analytic subgroup of $G$ having $\mathfrak{h}$ as its Lie algebra.

Theorem 1.3 (Locally Splitting Lie Group Quotients). Assume, firstly, that $G$ is a proLie group and that $N$ is a normal subgroup of $G$ such that $G / N$ is a Lie group.

Assume, secondly, that the Lie algebra $\mathfrak{g}$ of $G$ is the semidirect product of the Lie algebra $\mathrm{n}$ of $N$ and some closed subalgebra of $\mathfrak{g}$.

Let $\mu: N \rtimes_{\alpha} \widetilde{G / N} \rightarrow G$ be the morphism defined by $\mu(n, h)=n \varphi(h), \varphi: \widetilde{G / N} \rightarrow G$, as in Proposition 1.1.

Then the morphism $\mu$ is an open morphism with a kernel isomorphic to a discrete central subgroup of the Lie group $G / N$, and implements a local isomorphism between $N \rtimes_{\alpha} G / N$ and $G$.

If $\mathfrak{g}=\mathfrak{n} \oplus \mathfrak{h}$ semidirectly, then the analytic subgroup $A(\mathfrak{h})$ of $G$ having Lie algebra $\mathfrak{h}$ agrees with $\varphi(G / N)$, and has a countable intersection with $N$.

$N / N_{0}$ is locally compact metric totally disconnected and $N$ has an open almost connected subgroup $N_{1} \in \mathscr{N}(G)$ for which the same conclusions hold when $N$ is replaced by $N_{1}$.

Proof. Since $G / N$ is a Lie group, its identity component $(G / N)_{0}$ is open. There is therefore an open subgroup $\mathbb{G}$ of $G$ containing $N$ such that $\mathbb{G} / N=(G / N)_{0}$. We notice that $\widetilde{G / N}=\widetilde{\mathbb{G} / N}$. We observe that if the assertions of the theorem are proved for $\mathbb{G}$ in place of $G$ then they hold for $G$ since $\mathbb{G}$ is open in $G$. For the purposes of the proof we may therefore simplify notation by assuming that $G / N$ is connected, which we shall do henceforth.

Now we must verify the hypotheses of 1.2. Firstly, the assumptions of 1.1 are satisfied. Next Hypothesis 1.2(v) is satisfied, since the universal morphism

$$
\pi_{G / N}: \widetilde{G / N} \rightarrow G / N
$$

of a Lie group is none other than the universal covering morphism which is surjective. Secondly, the kernel $\operatorname{ker} \mu$ of $\mu$ is isomorphic to a totally disconnected central subgroup of the Lie group $\widehat{G / N}$ by 1.1 (iii) and is therefore discrete. By 1.1(iv), $N \rtimes_{\alpha} \widetilde{G / N}$ is a pro-Lie group. Then the quotient $\left(N \rtimes_{\alpha} \widetilde{G / N}\right) / \operatorname{ker} \mu$ is complete since any quotient of a Hausdorff topological groups modulo a first countable closed or locally compact normal subgroup is complete: see for instance [12], p. 242, Lemma 13.13, and [12], p. 206, Theorem 11.18. Thus 1.2(vi) is satisfied as well. Now 1.2 
applies and shows that the morphism $\mu$ is a quotient morphism and that its kernel is a discrete subgroup; it therefore implements a local isomorphism.

The subgroup

$$
\frac{(\{1\} \times H) \operatorname{ker} \mu}{\operatorname{ker} \mu}=\frac{(N \cap \varphi(H)) \times H}{\left\{\left(\varphi(h)^{-1}, h\right): h \in \varphi^{-1}(N)\right\}} \cong \frac{H}{\varphi^{-1}(N)}
$$

is mapped bijectively onto the analytic subgroup $A(\mathfrak{h})$ of $G$ with Lie algebra $\mathfrak{h}$. It intersects $N$ in a countable subgroup since the subgroup ker $\pi_{G / N}$ of $\widetilde{G / N}$ is finitely generated abelian and thus is countable.

By Definition 9.43 and Theorem 9.44 of [9], the factor group $G / N_{0}$ is finitedimensional, hence locally compact metric. And it is no loss of generality that $N / N_{0}$ corresponds to $\Delta$ in 9.44 of [9]. Therefore we may assume that $N$ is almost connected if we wish.

Corollary 1.4. If, in the circumstances of Theorem 1.3, the sum $\mathfrak{g}=\mathfrak{n} \oplus \mathfrak{h}$ is direct with an ideal $\mathfrak{h}$, then there is a direct product $N \times \widetilde{G / N}$ and an open morphism $\mu: N \times \widetilde{G / N} \rightarrow G$ with a discrete kernel implementing a local isomorphism. In particular, $G$ is locally isomorphic to the direct product of $N$ and the Lie group $G / N$.

Proof. We continue to write $H$ and again assume without loss of generality that $G / N$ is connected. The closed connected subgroup $\varphi(H)$ is the minimal analytic subgroup with Lie algebra $\mathfrak{h}$ and is closed. Now $\mathfrak{h}$ is not only a subalgebra, but an ideal. This implies that $\varphi(H)$ is normal. Since $N$ is normal, $[N, \varphi(H)] \subseteq N \cap \varphi(H)$. Since $\varphi(H)$ is connected, $[N, \varphi(H)]$ is connected and contains 1 . On the other hand, $N \cap \varphi(H)$ is a countable Hausdorff topological group and is therefore totally disconnected. It follows that $[N, \varphi(H)]$ is singleton and thus $N$ and $\varphi(H)$ commute elementwise. Thus $\alpha: H \rightarrow \operatorname{Aut}(N), \alpha(h)(n)=\varphi(h) n \varphi(h)^{-1}=n$ is the constant morphism. Therefore $N \rtimes_{\alpha} H$ is a direct product.

With these results we are now poised to shift the emphasis of our research to the Lie algebra.

\section{The Lie Algebra Theory of the Local Splitting}

The following definitions are put into place mainly for the purpose of convenience in the formulations that follow.

Definition 2.1. An ideal $\mathrm{n}$ in a pro-Lie algebra is called complemented, respectively, well-complemented if there a finite-dimensional subalgebra $\mathfrak{h}$ such that $\mathfrak{g}$ is the semidirect sum, respectively, direct sum, $\mathfrak{n} \oplus \mathfrak{h}$ algebraically and topologically. It is called supplemented if there is a finite-dimensional subalgebra $\mathfrak{h}$ such that $\mathfrak{g}=\mathfrak{n}+\mathfrak{h}$. 
Every well-complemented ideal is complemented, and every complemented ideal is supplemented. Every supplemented ideal $\mathfrak{n}$ is cofinite-dimensional, that is, $\operatorname{dim} \mathfrak{g} / \mathfrak{n}<\infty$.

Remember that the essential structural ingredient and invariant of a pro-Lie algebra $\mathfrak{g}$ is the filter basis $\mathscr{I}(\mathfrak{g})$ of all cofinite dimensional closed ideals $\mathfrak{i}$, and recall that $\mathscr{I}(\mathfrak{g})$ converges to zero. So supplemented ideals always are elements of $\mathscr{I}(\mathfrak{g})$.

Definition 2.2. A pro-Lie algebra $\mathfrak{g}$ is said to be rich, meaning "rich in complemented ideals," if $\mathfrak{g}$ has arbitrarily small complemented ideals; that is, for every zero neighborhood $U$ of $\mathfrak{g}$ there is a complemented ideal $\mathfrak{n}$ contained in $U$. It is called very rich if $\mathfrak{g}$ has arbitrarily small well-complemented ideals. Finally $\mathfrak{g}$ is said to be extremely rich if all sufficiently small ideals are well-complemented.

Notice that the set $\mathscr{C} \mathscr{I}(\mathfrak{g})$ of complemented ideals of a pro-Lie algebra contains $\mathfrak{g}$ and thus is not empty. Therefore, an alternative way of expressing the condition of richness is saying that

$\mathfrak{g}$ is rich iff $\mathscr{C} \mathscr{I}(\mathfrak{g})$ is cofinal in $\mathscr{I}(\mathfrak{g})$.

Every finite-dimensional Lie algebra is extremely rich by default since $\{0\}$ is wellcomplemented in this case.

We recall at this point that a pro-Lie algebra is called reductive if $\mathfrak{g}=\prod_{\mathfrak{i} \in J} \mathfrak{s} j$ for a family of finite-dimensional simple Lie algebras $\mathfrak{s}_{j}$ or 1-dimensional algebras isomorphic to $\mathbb{R}$. (See [9], Chapter 7, notably, Theorem 7.27.)

\section{Proposition 2.3.}

(i) Any ideal of a reductive pro-Lie algebra is a direct Lie algebra summand algebraically and topologically.

(ii) Every reductive pro-Lie algebra is extremely rich.

Proof. (i) follows from Theorem 7.27(a) of [9].

(ii) By definition of the product topology, given a zero neighborhood $U$, there is a finite subset $F \subseteq J$ and a family of zero neighborhoods $U_{j} \subseteq \mathfrak{s}_{j}$ for $j \in F$ such that $\prod_{j \in J} W_{j}$ is a zero neighborhood contained in $U$ if

$$
W_{j}= \begin{cases}\mathfrak{s}_{j} & \text { for } j \in J \backslash F \\ U_{j} & \text { for } j \in F\end{cases}
$$

provided the finitely many $U_{j}, j \in F$ are selected small enough. Now for this particular set $F$ we construct $\mathfrak{n}$ as in (i) above. Then we indeed have $\mathfrak{n} \subseteq \prod_{j \in J} W_{j} \subseteq U$ and $\mathrm{n}$ is well-complemented as we saw in (i).

A bit more generally, we can formulate the following result: 
Proposition 2.4. An arbitrary product of finite dimensional Lie algebras is very rich.

Proof. By following the line of argument of the proof of Proposition 2.3, the proof follows straightforwardly.

It is important that we take note of some examples which show that not every pro-Lie algebra is rich, let alone very rich. We shall use some multilinear algebra for weakly complete topological vector spaces which is provided in [9], Appendix 2, notably in Lemma A2.21, Lemma A2.22, and Corollary A2.23, or in [2], Chapter 7, Definition $7.27 \mathrm{ff}$.

Examples 2.5. (i) We write $\mathfrak{g}=\mathbb{R}^{\mathbb{N}_{0}}$, where $\mathbb{N}_{0}=\{0,1,2, \ldots\}$; and for any subset $J \subseteq \mathbb{N}_{0}$ we identify $\mathbb{R}^{J}$ with the obvious vector subspace of $\mathfrak{g}$. The bracket operation of $\mathfrak{g}$ is written

$$
\begin{aligned}
{\left[\left(x, r_{1}, r_{2}, \ldots\right),\left(y, s_{1}, s_{2}, \ldots\right)\right] } & =\left(x \cdot\left(0,0, s_{1}, s_{2}, \ldots\right)-y \cdot\left(0,0, r_{1}, r_{2}, \ldots\right)\right) \\
& =\left(0,0, x s_{1}-y r_{1}, x s_{2}-y r_{2}, \ldots\right)
\end{aligned}
$$

Then $\mathfrak{g}$ has a descending sequence of ideals $\mathfrak{g}^{[n]}=\mathbb{R}^{\{n+1, n+2, \ldots\}}, n=1,2, \ldots$, each of codimension one in the preceding one, but none of them is supplemented, let alone complemented. Thus $\mathfrak{g}$ is a pronilpotent (see [9], 7.42) center-free algebra which is not rich.

(ii) For $n \in \mathbb{N}$, let $\mathbb{C}_{n}$ be the $\mathbb{R}$-module defined on the underlying real vector space of $\mathbb{C}$ defined by the action $(r, c) \mapsto r{ }_{n} c=2 \pi n i r c: \mathbb{R} \times \mathbb{C} \rightarrow \mathbb{C}$. This is the Lie algebra action corresponding to the group action of the circle group $\mathbb{R} / \mathbb{Z}$ given by $(r+\mathbb{Z}, c) \mapsto e^{2 \pi i n r} c: \mathbb{R} / \mathbb{Z} \times \mathbb{C} \rightarrow \mathbb{C}$. We consider the product module $V \stackrel{\text { def }}{=} \prod_{n \in Z} \mathbb{C}_{n}$ with the morphism $\alpha: \mathbb{R} \rightarrow \operatorname{Der}(V)$ given by $\alpha(r)\left(c_{n}\right)_{n \in \mathbb{Z}}=\left(r \cdot_{n} c_{n}\right)_{n \in \mathbb{Z}}$. Now we form the semidirect product $\mathfrak{g} \stackrel{\text { def }}{=} V \rtimes_{\alpha} \mathbb{R}$, that is, the product $V \times \mathbb{R}$ with componentwise addition and the bracket $[(c, r),(d, s)]=(\alpha(r)(d)-\alpha(s)(c), 0)$.

Then $\mathfrak{g}$ is a metabelian rich pro-Lie algebra that is not very rich.

(iii) This example will present the pro-Heisenberg algebra $\mathfrak{h}(V)$ over a given weakly complete topological vector space $V$ with $\operatorname{dim} V>1$. We define

$$
\mathfrak{h}(V)=V \times \bigwedge^{2} V, \quad[(v, x),(w, y)]=(0, v \wedge w),
$$

with the componentwise topological vector space structure. Then $\operatorname{dim} \geq 2$ implies that $\mathfrak{h}(V)$ is a class 2 nilpotent pro-Lie algebra, and $\overline{[\mathfrak{h}(V), \mathfrak{h}(V)]}=\mathfrak{z}(\mathfrak{h}(V))=$ $\{0\} \times \bigwedge^{2} V$ since the span of the $v \wedge w$ is dense in $\bigwedge^{2} V$. We record that $\mathfrak{g} \stackrel{\text { def }}{=} \mathfrak{h}(V)$ is its own nilradical $\mathfrak{n}(\mathfrak{g})$ and $\mathfrak{n}(\mathfrak{g}) / \mathfrak{z}(\mathfrak{g}) \cong V$.

Now assume that $\mathfrak{g} \stackrel{\text { def }}{=} \mathfrak{h}(V)$ is an ideal direct sum $\mathfrak{g}_{1} \oplus \mathfrak{g}_{2}$. Assume $\mathfrak{g}_{1} \subseteq \mathfrak{z}(\mathfrak{g})$, then $\mathfrak{z}(\mathfrak{g})=\overline{\left[\mathfrak{g}_{1}+\mathfrak{g}_{2}, \mathfrak{g}_{1}+\mathfrak{g}_{2}\right]}=\overline{\left[\mathfrak{g}_{2}, \mathfrak{g}_{2}\right]} \subseteq \mathfrak{g}_{2}$ and so $\mathfrak{g}_{1} \subseteq \mathfrak{g}_{1} \cap \mathfrak{g}_{2}=\{0\}$. Thus suppose that neither of the summands is zero. Then none of them is contained in $\mathfrak{z}(\mathfrak{g})$. Let $\left(v_{1}, z_{1}\right) \in \mathfrak{g}_{1} \backslash \mathfrak{z}(\mathfrak{g})$ and $\left(v_{2}, z_{2}\right) \in \mathfrak{g}_{2} \backslash \mathfrak{z}(\mathfrak{g})$. Then $(0,0)=\left[\left(v_{1}, z_{1}\right),\left(v_{2}, z_{2}\right)\right]=\left(0, v_{1} \wedge v_{2}\right)$. 
Thus $v_{2} \in \mathbb{R} \cdot v_{1}$ by Corollary $\mathrm{A} 2.23$ of [9], that is, there is a nonzero $r \in \mathbb{R}$ such that $v_{2}=r \cdot v_{1}$, and so $v_{1} \wedge V=v_{2} \wedge V \neq\{0\}$. Since $\mathfrak{g}_{1}$ and $\mathfrak{g}_{2}$ are ideals, we have $\{0\} \times\left(v_{1} \wedge V\right)=\left[\left(v_{1}, z_{1}\right), \mathfrak{g}\right] \subseteq \mathfrak{g}_{1}$ and $\{0\} \times\left(v_{2} \wedge V\right)=\left[\left(v_{2}, z_{2}\right), \mathfrak{g}\right] \subseteq \mathfrak{g}_{2}$. Hence $\{(0,0)\} \neq\{0\} \times\left(v_{1} \wedge V\right) \subseteq \mathfrak{g}_{1} \cap \mathfrak{g}_{2}$, and this is a contradiction. Thus $\mathfrak{h}(\mathfrak{g})$ does not allow an ideal direct sum decomposition.

In particular, if $\operatorname{dim} V=\infty$, then $\mathfrak{h}(V)$ is a nilpotent class 2 pro-Lie algebra that is not very rich. On the other hand, if $V=\operatorname{span}\left(\left\{e_{1}, e_{2}\right\}\right)$, then $V$ is the semidirect product of the ideal $\mathfrak{z}(\mathfrak{h}(V)) \oplus \mathbb{R} \cdot\left(e_{1} .0\right)=\mathbb{R} \cdot e_{1} \times \mathbb{R} \cdot\left(e_{1} \wedge e_{2}\right)$ and the subalgebra $\mathbb{R} \cdot\left(e_{2}, 0\right)$.

The Campbell-Hausdorff multiplication on $\mathfrak{h}(\mathfrak{g})$ is given by $(v, x) *(w, y)=(v+w$, $\left.x+y+\frac{1}{2}(v \wedge w)\right)$. The pro-Lie group $(\mathfrak{h}(\mathfrak{g}), *)$ is called the pro-Heisenberg group $H(V)$ over the weakly complete topological vector space $V$. If $\operatorname{dim} V$ is infinite, $H(V)$ is an example of a nilpotent pro-Lie group of class 2 which does not satisfy Iwasawa's Local Splitting Theorem.

[For a verification of the details of Example 2.5(i), for a finite subset $F \subseteq \mathbb{Z}$, identify $V_{F}=\prod_{n \in F} \mathbb{C}_{n}$ in the obvious way with a subgroup of $V$. Show that $\left(V_{\mathbb{Z} \backslash F} \times\{0\}\right) \rtimes$ $\left(V_{F} \times \mathbb{R}\right)$ with the action of $V_{F} \times \mathbb{R}$ on $V_{\mathbb{Z} \backslash F} \times\{0\}$ via $\mathbb{R}$. Show that a direct product decomposition as required in a rich algebra is not possible.]

If $\operatorname{dim} V=2$, then $H(V)^{\mathbb{N}}$ is a class 2 nilpotent pro-Lie group satisfying the Iwasawa Local Splitting Theorem.

Every pro-Lie algebra $\mathfrak{g}$ contains a unique smallest ideal $\mathfrak{n}_{\text {cored }}(\mathfrak{g})$ such that $\mathfrak{g} / \mathfrak{n}_{\text {cored }}(\mathfrak{g})$ is reductive; moreover $\mathfrak{n}_{\text {cored }}(\mathfrak{g})$ is pronilpotent and equals $\overline{[\mathfrak{g}, \mathfrak{r}(\mathfrak{g})]}$ for the radial $\mathfrak{r}(\mathfrak{g})$ of g. (See [9], Definition 7.65 and Theorems 7.66, 7.67). We call $n_{\text {cored }}(\mathfrak{g})$ the coreductive radical of $\mathfrak{g}$.

Lemma 2.6. Let $\mathfrak{g}$ be a pro-Lie algebra.

(i) Assume that $\mathfrak{\mathfrak { }}$ is a finite-dimensional ideal such that $\mathfrak{g} / \mathfrak{f}$ is rich, respectively, very rich. Then $\mathfrak{g}$ is rich, respectively, very rich. Indeed there are arbitrarily small ideals $\mathfrak{i} \in \mathscr{I}(\mathfrak{g})$ for which there is a finite-dimensional subalgebra, respectively, ideal $\mathfrak{h}$ containing $\mathfrak{\mathfrak { f }}$ such that $\mathfrak{g}=\mathfrak{i} \oplus \mathfrak{h}$.

(ii) Assume that $\operatorname{dim} \mathfrak{n}_{\text {cored }}(\mathfrak{g})<\infty$. Then $\mathfrak{g}$ is very rich.

Proof. (i) Since $\operatorname{dim} \mathfrak{f}<\infty$ and $\lim \mathscr{I}(\mathfrak{g})=0$ we find a $\mathrm{i}_{0} \in \mathscr{I}(\mathfrak{g})$ such that $\mathrm{i}_{0} \cap$ $\mathfrak{f}=\{0\}$. Then $\mathfrak{j} \in \mathscr{I}(\mathfrak{g})$ and $\mathfrak{j} \subseteq \mathfrak{j}_{0}$ implies $\mathfrak{j} \cap \mathfrak{f}=\{0\}$. Since $\mathfrak{f}$ is finite-dimensional, $\dot{\mathrm{j}}_{0}+\mathfrak{f}$ is closed and since $\mathfrak{f}$ is an ideal, $\dot{\mathrm{j}}_{0} \oplus \mathfrak{f}$ is an ideal direct sum and $\left(\mathfrak{j}_{0} \oplus \mathfrak{f}\right) / \tilde{\mathfrak{T}} \in$ $\mathscr{I}(\mathfrak{g} / \mathfrak{f})$. Since $\mathfrak{g} / \mathfrak{f}$ is rich, there is an ideal $\mathfrak{i}$ of $\mathfrak{g}$ containing $\mathfrak{f}$ such that $\mathfrak{i} \subseteq \mathfrak{i}_{0} \oplus \mathfrak{f}$ and that $\mathfrak{g} / \mathfrak{f}$ is a semidirect sum $\mathfrak{i} / \mathfrak{f} \oplus \mathfrak{h} / \mathfrak{f}$ for a subalgebra $\mathfrak{h}$ of $\mathfrak{g}$ such that $\operatorname{dim} \mathfrak{h} / \mathfrak{f}<\infty$, implying that $\operatorname{dim} \mathfrak{h}<\infty$. So $\mathfrak{g}=\mathfrak{i}+\mathfrak{h}$ and $\mathfrak{i} \cap \mathfrak{h}=\mathfrak{f}$ while, on the other hand, $\mathfrak{f} \subseteq$ $\mathfrak{i} \subseteq \mathfrak{j}_{0} \oplus \mathfrak{f}$. Then the modular law implies $\mathfrak{i}=\left(\mathfrak{i} \cap \dot{j}_{0}\right) \oplus \mathfrak{f}$. Therefore $\mathfrak{g}=\mathfrak{i}+\mathfrak{h}=$ $\left(\mathfrak{i} \cap \dot{j}_{0}\right) \oplus \mathfrak{f}+\mathfrak{h}=\left(\mathfrak{i} \cap \dot{j}_{0}\right)+\mathfrak{h}$ and $\mathfrak{i} \cap \dot{j}_{0} \cap \mathfrak{h}=\dot{\mathfrak{j}}_{0} \cap \mathfrak{f}=\{0\}$. Thus, since $\mathfrak{h}$ is finite- 
dimensional, $\mathfrak{g}$ is the semidirect sum $\left(\mathfrak{i} \cap \dot{j}_{0}\right) \oplus \mathfrak{h}$ with $\mathfrak{i} \cap \dot{j}_{0} \in \mathscr{I}(\mathfrak{g})$. Since $\dot{j}_{0}$ may be taken arbitrarily small, this shows that $\mathfrak{g}$ is rich. If $\mathfrak{g} / \mathfrak{f}$ is very rich, $\mathfrak{h}$ may be chosen to be an ideal, and so the sum $(\mathfrak{i} \cap \mathfrak{i}) \oplus \mathfrak{h}$ is direct, showing that $\mathfrak{g}$ is very rich. If $\mathfrak{g} / \mathfrak{f}$ is extremely rich, the argument works for all sufficiently small $\mathfrak{i} \in \mathscr{I}(\mathfrak{g})$, and this shows that $\mathfrak{g}$ is extremely rich.

(ii) is an immediate consequence of (i).

Proposition 2.7. Let $\mathfrak{g}$ be a pro-Lie algebra, $\mathfrak{g}^{\prime}=[\mathfrak{g}, \mathfrak{g}]$ its commutator algebra, and $\mathfrak{z}(\mathfrak{g})$ its center. Then the following statements are equivalent:
(A) $\operatorname{dim} \mathfrak{g}^{\prime}<\infty$,
(B) $\quad \operatorname{dim} \mathfrak{g} / \mathfrak{\jmath}(\mathfrak{g})<\infty$.

If these conditions are satisfied, then $\mathfrak{g}^{\prime}$ is finite-dimensional, and therefore closed. Let $\mathfrak{z} \subseteq \mathfrak{z}(\mathfrak{g})$ be a cofinite-dimensional closed vector subspace and $\mathfrak{v}$ be a finite-dimensional vector subspace such that $\mathfrak{g}=\mathfrak{z} \oplus \mathfrak{v}$.

(i) $\mathfrak{\mathfrak { f }}=\mathfrak{g}^{\prime} \oplus \mathfrak{v}$ is a finite-dimensional ideal containing $\mathfrak{g}^{\prime}$ and satisfying $\mathfrak{g}=\mathfrak{z}+\mathfrak{f}$. In particular, there is a central ideal $\mathfrak{a} \in \mathscr{I}(\mathfrak{g})$ such that $\mathfrak{g}=\mathfrak{a} \oplus \mathfrak{f}$ and $\mathfrak{a} \subseteq \mathfrak{z}$.

(ii) $\mathfrak{g}$ is very rich.

Proof. $(\mathrm{B}) \Rightarrow(\mathrm{A})$ : Write $\mathfrak{g}=\mathfrak{z}(\mathfrak{g}) \oplus \mathfrak{v}$ with a finite-dimensional vector subspace $\mathfrak{v}$. Then $\mathfrak{g}^{\prime} \stackrel{\text { def }}{=}[\mathfrak{g}, \mathfrak{g}]=\operatorname{span}\left\{\left[z+v, z^{\prime}+v^{\prime}\right]: z, z^{\prime} \in \mathfrak{z}(\mathfrak{g}), v, v^{\prime} \in \mathfrak{v}\right\}=\operatorname{span}\left\{\left[v, v^{\prime}\right]: v, v^{\prime} \in \mathfrak{v}\right\}$ is finite-dimensional since $\mathfrak{v}$ is finite-dimensional.

$(\mathrm{A}) \Rightarrow(\mathrm{B})$ : Assume that $\operatorname{dim} \mathfrak{g}^{\prime}<\infty$. Denote by $b: \mathfrak{g} \times \mathfrak{g} \rightarrow \mathfrak{g}^{\prime}$ the continuous bilinear map given by $b(x, y)=[x, y]$. Then it follows from Lemma A2.21 of Appendix 2 of [9] that there is a cofinite-dimensional closed vector subspaces $E$ of $\mathfrak{g}$ such that $[E, \mathfrak{g}]=\{0\}$ and that therefore $E \subseteq \mathfrak{z}(\mathfrak{g})$. Thus $\mathfrak{z}(\mathfrak{g})$ is cofinite-dimensional as asserted.

(i) We note that $\mathfrak{f}$ contains $\mathfrak{g}^{\prime}$ and recall that every vector subspace of a Lie algebra $\mathfrak{g}$ containing $\mathfrak{g}^{\prime}$ is an ideal. We find a closed vector subspace $\mathfrak{a}$ of $z$ such that $\mathfrak{z}=\mathfrak{a} \oplus(\mathfrak{z} \cap \mathfrak{f})$. Then $\mathfrak{g}=\mathfrak{a} \oplus \mathfrak{f}$ is a direct sum of pro-Lie algebras and $\mathfrak{a} \subseteq \mathfrak{z} \subseteq \mathfrak{z}(\mathfrak{g})$.

(ii) This follows from (i) and Lemma 2.6. (It is also immediate from (ii) directly.)

The implication $(\mathrm{B}) \Rightarrow(\mathrm{A})$ is always true while the implication $(\mathrm{A}) \Rightarrow(\mathrm{B})$ fails in general as is shown by the example $\mathfrak{g}=\mathbb{R}^{(\mathbb{N})} \times \mathbb{R}$ with the bracket

$$
\left[\left(\sum_{n=1}^{\infty} u_{n}, r\right),\left(\sum_{n=1}^{\infty} v_{n}, s\right)\right]=\left(0, \sum_{n=1}^{\infty} \operatorname{det}\left(u_{n}, v_{n}\right)\right)
$$

Here $\{0\} \times \mathbb{R}$ is $\mathfrak{g}^{\prime}$ and $\mathfrak{z}(\mathfrak{g})$ at the same time, and the center therefore is far from being cofinite-dimensional.

As an exercise, we verify the following 
Remark. (i) Let $\mathfrak{g}$ be a pro-Lie algebra and $\mathfrak{z}$ an ideal contained in the center $\mathfrak{z}(\mathfrak{g})$ of $\mathfrak{g}$. If $\mathfrak{g} / \mathfrak{z}$ is pronilpotent, then $\mathfrak{g}$ is pronilpotent.

(ii) The nilradical $\mathfrak{n}(\mathfrak{g} / \mathfrak{z}(\mathfrak{g}))$ of $\mathfrak{g} / \mathfrak{z}(\mathfrak{g})$ is $\mathfrak{n}(\mathfrak{g}) / \mathfrak{z}(\mathfrak{g})$.

[Outline of proof. (i) Let $\mathrm{i} \in \mathscr{I}(\mathfrak{g})$. Then $(\mathfrak{g} / \mathrm{i}) /((\mathfrak{z}+\mathrm{i}) / \mathrm{i}) \cong \mathfrak{g} /(\mathfrak{z}+\mathrm{i})$ is a finitedimensional quotient of $\mathfrak{g} / \mathfrak{z}$ and is, therefore, a nilpotent Lie algebra. Also, $(\mathfrak{z}+\mathrm{i}) / \mathrm{i}$ is contained in the center of $\mathfrak{g} / \mathfrak{i}$. Therefore $\mathfrak{g} / \mathfrak{i}$ is nilpotent. Thus $\mathfrak{g}$ is pronilpotent.

(ii) Let $\mathfrak{m}$ be that ideal of $\mathfrak{g}$ containing $\mathfrak{z}(\mathfrak{g})$ for which $\mathfrak{m} / \mathfrak{z}(\mathfrak{g})=\mathfrak{n}(\mathfrak{g} / \mathfrak{z}(\mathfrak{g}))$. Since this quotient is pronilpotent, by (i) above we know that $\mathrm{m}$ is pronilpotent. Hence $\mathfrak{m} \subseteq \mathfrak{n}(\mathfrak{g})$. Since $\mathfrak{n}(\mathfrak{g}) / \mathfrak{z}(\mathfrak{g})$ is pronilpotent, by Lemma 7.56(ii) and Theorem 7.57 of [9], we have $\mathfrak{n}(\mathfrak{g}) \subseteq \mathfrak{m}$. Thus $\mathfrak{m}=\mathfrak{n}(\mathfrak{g})$.]

Lemma 2.8. (i) Let $p: \mathfrak{h} \rightarrow \mathfrak{f}$ be a surjective morphism of pro-Lie algebras. Then $p\left(\mathfrak{n}_{\text {cored }}(h)\right)=\mathfrak{n}_{\text {cored }}(\mathfrak{f})$, and if $\operatorname{ker} p \subseteq \mathfrak{z}(\mathfrak{h})$, then $p(\mathfrak{n}(\mathfrak{h}))=\mathfrak{n}(\mathfrak{f})$.

(ii) Let $\mathfrak{j}$ be an ideal of a pro-Lie algebra $\mathfrak{g}$ and assume that it contains the center. Then $\mathfrak{n}(\mathfrak{i}) / \mathfrak{z}(\mathfrak{i})$ is a quotient of an ideal of $\mathfrak{n}(\mathfrak{g}) / \mathfrak{z}(\mathfrak{g})$. In particular, if $\mathfrak{n}(\mathfrak{g}) / \mathfrak{z}(\mathfrak{g})$ is finitedimensional, so is $\mathfrak{n}(\mathrm{i}) / \mathfrak{z}(\mathrm{i})$.

Proof. (i) Let $p: \mathfrak{h} \rightarrow \mathfrak{f}$ be a surjective morphism of pro-Lie algebras. Then $p(\mathfrak{r}(\mathfrak{h}))=\mathfrak{r}(\mathfrak{f})$ by Proposition 7.54 of [9]. Thus $p$ maps $[\mathfrak{h}, \mathfrak{r}(\mathfrak{h})]$ onto $[\mathfrak{f}, \mathfrak{r}(\mathfrak{f})]$. The image of $[\mathfrak{h}, \mathfrak{r}(\mathfrak{h})]$ by $p$ is closed according to Theorem A2.12(b) in Appendix 2 of [9] or [4], Proposition 3.1. Hence, in view of Theorem 7.67 of [9], we get $p\left(\mathfrak{n}_{\text {cored }}(\mathfrak{b})\right)=$ $p(\overline{[\mathfrak{h}, \mathfrak{r}(\mathfrak{h})]})=\overline{[\mathfrak{f}, \mathfrak{r}(\mathfrak{f})]}=\mathfrak{n}_{\text {cored }}(\mathfrak{f})$. Moreover, $p(\mathfrak{n}(\mathfrak{h}))$ is a pronilpotent ideal and therefore is contained in $\mathfrak{n}(\mathfrak{f})$. Now assume that ker $p$ is central; then $p^{-1}(\mathfrak{n}(\mathfrak{f})) / \mathfrak{z}(\mathfrak{h}) \cong \mathfrak{n}(\mathfrak{f})$ is pronilpotent and thus $p^{-1}(\mathfrak{n}(\mathfrak{f}))$ is pronilpotent by the Remark preceding this Lemma.

(ii) According to (i), the quotient map $\mathrm{i} / \mathfrak{\jmath}(\mathfrak{g}) \rightarrow \mathrm{i} / \mathfrak{z}(\mathrm{i})$ maps $\mathfrak{n}(\mathrm{i}) / \mathfrak{z}(\mathfrak{g})$ onto $\mathfrak{n}(\mathrm{i}) / \mathfrak{z}(\mathrm{i})$. Since $\mathfrak{n}(\mathfrak{i})$ as a characteristic ideal of $\mathfrak{j}$ is a pronilpotent ideal of $\mathfrak{g}$, we have $\mathfrak{n}(\mathfrak{i}) \subseteq \mathfrak{n}(\mathfrak{g})$ and thus $\mathfrak{n}(\mathfrak{i}) / \mathfrak{z}(\mathfrak{g}) \subseteq \mathfrak{n}(\mathfrak{g}) / \mathfrak{z}(\mathfrak{g})$. This proves the first assertion of (ii), and the second is an immediate consequence.

If we apply 2.8(i) to the quotient morphism

$$
p: \mathfrak{g} \rightarrow \mathfrak{g} / \mathfrak{\jmath}(\mathfrak{g}),
$$

we obtain

$$
\left(\mathfrak{n}_{\text {cored }}(\mathfrak{g})+\mathfrak{\jmath}(\mathfrak{g})\right) / \mathfrak{\jmath}(\mathfrak{g})=\mathfrak{n}_{\text {cored }}(\mathfrak{g} / \mathfrak{\jmath}(\mathfrak{g}))
$$

and so the full inverse image $p^{-1}\left(\mathfrak{n}_{\text {cored }}(\mathfrak{g} / \mathfrak{\jmath}(\mathfrak{g}))\right.$ agrees with $\mathfrak{n}_{\text {cored }}(\mathfrak{g})+\mathfrak{\jmath}(\mathfrak{g})$.

Definition 2.9. The pronilpotent pro-Lie algebra $\mathfrak{n}(\mathfrak{g}) / \mathfrak{z}(\mathfrak{g}))=\mathfrak{n}(\mathfrak{g} / \mathfrak{z}(\mathfrak{g}))$ is called the nilcore of $\mathfrak{g}$. It is written nilcore $(\mathfrak{g})$. 


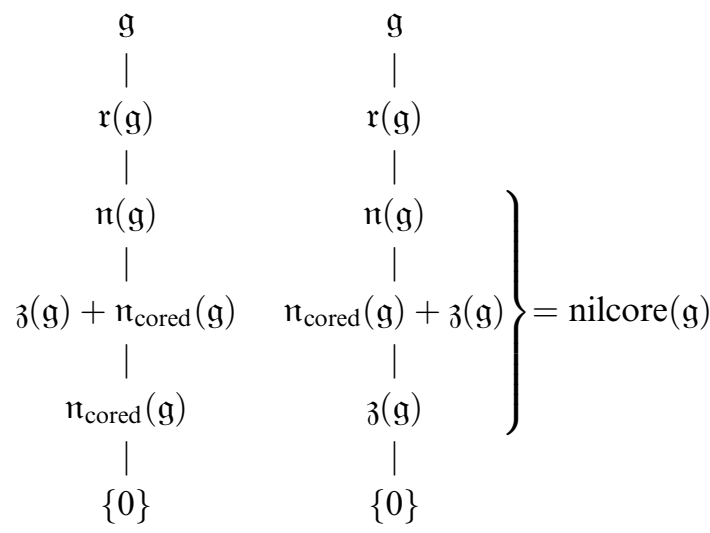

We recall the following elementary examples showing that all containments are proper in general. In the Heisenberg algebra $\operatorname{span}\{X, Y, Z\},[X, Y]=Z$, we have

$$
\mathfrak{z}(\mathfrak{g})=\mathfrak{n}_{\text {cored }}(\mathfrak{g})=\mathfrak{z}(\mathfrak{g})+\mathfrak{n}_{\text {cored }}(\mathfrak{g})=\mathbb{R} \cdot Z \neq \mathfrak{g}=\mathfrak{r}(\mathfrak{g})=\mathfrak{n}(\mathfrak{g}) ;
$$

in the motion algebra $\operatorname{span}\{X, Y, Z\},[X, Y]=Z,[X, Z]=-Y$,

$$
\{0\}=\mathfrak{z}(\mathfrak{g}) \neq \mathfrak{n}_{\text {cored }}(\mathfrak{g})=\operatorname{span}\{Y, Z\}=\mathfrak{z}(\mathfrak{g})+\mathfrak{n}_{\text {cored }}(\mathfrak{g})=\mathfrak{n}(\mathfrak{g}) \neq \mathfrak{r}(\mathfrak{g})=\mathfrak{g} ;
$$

in $\operatorname{span}\{U, X, Y, Z\},[U, X]=Y,[U, Y]=Z$ we have

$$
\begin{aligned}
\{0\} & \neq \mathfrak{z}(\mathfrak{g})=\mathbb{R} \cdot Z \neq \mathfrak{n}_{\text {cored }}(\mathfrak{g})=\mathfrak{z}(\mathfrak{g})+\mathfrak{n}_{\text {cored }}(\mathfrak{g}) \\
& =\operatorname{span}\{X, Y, Z\} \neq \mathfrak{n}(\mathfrak{g})=\mathfrak{g} ;
\end{aligned}
$$

in the direct sum of the motion algebra with $\mathbb{R}$ we have

$$
\mathfrak{z}(\mathfrak{g}) \nsubseteq \mathfrak{n}_{\text {cored }}(\mathfrak{g}) ;
$$

in $\mathfrak{s o}(3)$ we have

$$
\mathfrak{r}(\mathfrak{g}) \neq \mathfrak{g} .
$$

In the sum of all of these, all of the containments in the tall Hasse diagram are proper. The following Lemma deals with the case that $\mathrm{n}_{\text {cored }}(\mathfrak{g}) \subseteq \mathfrak{z}(\mathfrak{g})$.

Lemma 2.10. Let $\mathfrak{g}$ be a pro-Lie algebra satisfying $\mathfrak{n}_{\text {cored }}(\mathfrak{g}) \subseteq \mathfrak{z}(\mathfrak{g})$. Then

(i) $\mathfrak{g}=\mathfrak{r}(\mathfrak{g}) \oplus \mathfrak{s}(\mathfrak{g})$ is a direct sum algebraically and topologically of the radical and $a$ unique Levi summand $\mathfrak{s}(\mathfrak{g})$.

(ii) $\overline{[\mathfrak{r}(\mathfrak{g}), \mathfrak{r}(\mathfrak{g})]}=\overline{[\mathfrak{g}, \mathfrak{r}(\mathfrak{g})]}=\mathfrak{n}_{\text {cored }}(\mathfrak{g}) \subseteq \mathfrak{z}(\mathfrak{g})$ and $\mathfrak{r}(\mathfrak{g})$ is nilpotent of class $\leq 2$, that is $\mathfrak{r}(\mathfrak{g})^{[2]}=[\mathfrak{r}(\mathfrak{g}),[\mathfrak{r}(\mathfrak{g}), \mathfrak{r}(\mathfrak{g})]]=\{0\}$. We have $\mathfrak{n}(\mathfrak{g})=\mathfrak{r}(\mathfrak{g})$. 
(iii) $\overline{[\mathfrak{g}, \mathfrak{g}]}=\overline{[\mathfrak{r}(\mathfrak{g}), \mathfrak{r}(\mathfrak{g})]} \oplus \mathfrak{s}(\mathfrak{g})$, and this is a reductive pro-Lie algebra. If $\mathfrak{a}$ is chosen so that $\mathfrak{z}(\mathfrak{g})=\mathfrak{n}_{\text {cored }}(\mathfrak{g}) \oplus \mathfrak{a}$, and $\mathfrak{r}(\mathfrak{g})=\mathfrak{z}(\mathfrak{g}) \oplus \mathfrak{v}$ for a closed vector subspace $\mathfrak{v}$ then $\mathfrak{n}_{\text {cored }}(\mathfrak{g}) \oplus \mathfrak{v}$ is a nilpotent ideal of class $\leq 2$ and $\mathfrak{g}=\mathfrak{s}(\mathfrak{g}) \oplus \mathfrak{a} \oplus \mathfrak{n}_{\text {cored }}(\mathfrak{g}) \oplus \mathfrak{v}$.

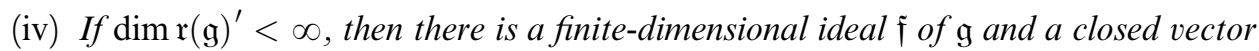
subspace a of $\mathfrak{z}(\mathfrak{g})$ such that $\mathfrak{g}$ is the ideal direct sum $\mathfrak{s}(\mathfrak{g}) \oplus \mathfrak{a} \oplus \mathfrak{\mathfrak { f }}$, and $\mathfrak{g}$ is very rich. If $\operatorname{dim}$ nilcore $(\mathfrak{g})<\infty$, then $\operatorname{dim} \mathfrak{r}(\mathfrak{g})^{\prime}<\infty$.

Proof. (i) By Corollary 7.75 and Theorem 7.77(i) of [9], two Levi summands are conjugate under an inner automorphism of the form $\alpha=e^{\operatorname{ad} x}$ for some $x \in \mathfrak{n}_{\text {cored }}(\mathfrak{g})$. By our hypothesis $x \in \mathfrak{n}_{\text {cored }}(\mathfrak{g})$ implies ad $x=0$ and thus $\alpha=\mathrm{id}_{\mathfrak{g}}$. Hence there is only one Levi summand. Thus, by Theorem 7.77(ii) of [9] we conclude (i).

(ii) From Theorem 7.67 of [9] we know that $\mathfrak{n}_{\text {cored }}(\mathfrak{g})=\overline{[\mathfrak{g}, \mathfrak{g}]} \cap \mathfrak{r}(\mathfrak{g})=\overline{[\mathfrak{g}, \mathfrak{r}(\mathfrak{g})]}$ and by hypothesis $n_{\text {cored }}(\mathfrak{g})$ is central. Conclusion (i) and Lemma 7.26 of [9] imply $\mathfrak{g}^{\prime}=[\mathfrak{g}, \mathfrak{g}]=\mathfrak{r}(\mathfrak{g})^{\prime}+\mathfrak{s}$. The factor algebra $\mathfrak{r}(\mathfrak{g}) / \mathfrak{z}(\mathfrak{g})$ is the center of $\mathfrak{g} / \mathfrak{z}(\mathfrak{g})$. Thus (ii) follows.

(iii) is now straightforward from (ii).

(iv) Since $[\mathfrak{r}(\mathfrak{g}), \mathfrak{s}(\mathfrak{g})]=\{0\}$ by (i), we have $\mathfrak{z}(\mathfrak{g})=\mathfrak{z}(\mathfrak{r}(\mathfrak{g}))$. Therefore, if $\mathfrak{r}(\mathfrak{g})^{\prime}$ is finite-dimensional, then Proposition 2.7 shows that $\mathfrak{r}(\mathfrak{g})=\mathfrak{a} \oplus \mathfrak{f}$ with a central ideal $\mathfrak{a}$ and a finite-dimensional ideal $\mathfrak{f}$ of $\mathfrak{r}(\mathfrak{g})$ and then also of $\mathfrak{g}$ by (i). Then (i) also implies $\mathfrak{g}=\mathfrak{s}(\mathfrak{g}) \oplus \mathfrak{a} \oplus \mathfrak{f}$. Since $\mathfrak{s}(\mathfrak{g}) \oplus \mathfrak{a}$ is reductive and $\operatorname{dim} \mathfrak{f}<\infty$, Proposition 2.3 and Lemma 2.6 imply that $\mathfrak{g}$ is very rich. The nilcore nilcore $(\mathfrak{g})$ is $\mathfrak{r}(\mathfrak{g}) / \mathfrak{z}(\mathfrak{g})$; so if this algebra is finite-dimensional then $\mathfrak{r}(\mathfrak{g})^{\prime}$ is finite-dimensional by 11.13 of [9].

Now we are ready for the structure theorem that is the Lie algebra nucleus of the local splitting theorems of this paper.

Theorem 2.11 (The Structure of Pro-Lie Algebras with Finite-Dimensional Nilcore). For a pro-Lie algebra $\mathfrak{g}$, the following two conditions are equivalent:

(i) The nilcore nilcore $(\mathfrak{g})=\mathfrak{n}(\mathfrak{g}) / \mathfrak{z}(\mathfrak{g})$ of $\mathfrak{g}$ is finite-dimensional.

(ii) $\mathfrak{g}$ is an ideal direct sum, algebraically and topologically, of a reductive pro-Lie algebra and a finite-dimensional Lie algebra.

If these conditions are satisfied, then all sufficiently small cofinite-dimensional ideals of $\mathfrak{g}$ are direct summands.

If the maximal compactly embedded ideal $\mathfrak{m}(\mathfrak{g})$ is cofinite dimensional in $\mathfrak{g}$ then $\operatorname{dim} \operatorname{nilcore}(\mathfrak{g})<\infty$.

Proof. Propositions 2.3 and Lemma 2.6 show that Condition (ii) implies that $\mathfrak{g}$ is very rich. If (ii) is satisfied, then $\mathfrak{g}$ is a direct sum $\mathfrak{g}_{1} \oplus \mathfrak{g}_{2}$ with a reductive ideal $\mathfrak{g}_{1}$ and a finite-dimensional ideal $\mathfrak{g}_{2}$. Then $\mathfrak{n}(\mathfrak{g})=\mathfrak{n}\left(\mathfrak{g}_{1}\right) \oplus \mathfrak{n}\left(\mathfrak{g}_{2}\right)$ and $\mathfrak{z}(\mathfrak{g})=\mathfrak{z}\left(\mathfrak{g}_{1}\right) \oplus \mathfrak{z}\left(\mathfrak{g}_{2}\right)$ and, accordingly, nilcore $(\mathfrak{g}) \cong \operatorname{nilcore}(\mathfrak{g})_{1} \times \operatorname{nilcore}(\mathfrak{g})_{2}$. But nilcore $(\mathfrak{g})_{1}=\{1\}$ and $\operatorname{dim} \mathfrak{g}_{2}<\infty$. Accordingly, dim nilcore $(\mathfrak{g})<\infty$.

By Corollary 12.34(iii) of [9], $\mathfrak{m}(\mathfrak{g}) \cap \mathfrak{r}(\mathfrak{g})=\mathfrak{z}(\mathfrak{g})$. Therefore, if $\mathfrak{g} / \mathfrak{m}(\mathfrak{g})$ is finite- 
dimensional, then $\mathfrak{r}(\mathfrak{g}) / \mathfrak{z}(\mathfrak{g})=\mathfrak{r}(\mathfrak{g}) /(\mathfrak{r}(\mathfrak{g}) \cap \mathfrak{m}(\mathfrak{g})) \cong(\mathfrak{r}(\mathfrak{g})+\mathfrak{m}(\mathfrak{g})) / \mathfrak{m}(\mathfrak{g})$ is finitedimensional and thus certainly $\mathfrak{n}(\mathfrak{g}) / \mathfrak{z}(\mathfrak{g})$ is finite-dimensional, that is, (i) holds.

It therefore remains to prove (i) $\Rightarrow$ (ii): By Lemma (ii) of the Remark preceding Lemma $2.8, \mathfrak{n}(\mathfrak{g} / \mathfrak{z}(\mathfrak{g}))=\mathfrak{n}(\mathfrak{g}) / \mathfrak{z}(\mathfrak{g})$. So, by hypothesis, $\mathfrak{g} / \mathfrak{z}(\mathfrak{g})$ has a finite-dimensional nilradical. Since the nilradical contains the coreductive radical (see Theorem 7.66ff. of [9]), $\mathfrak{g} / \mathfrak{n}(\mathfrak{g})$ is reductive. So $\mathfrak{g} / \mathfrak{n}(\mathfrak{g})$ is very rich by Proposition 2.3(ii). Thus Lemma 2.6(i) applies with $\mathfrak{g} / \mathfrak{z}(\mathfrak{g})$ in place of $\mathfrak{g}$ and with $\mathfrak{r}(\mathfrak{g}) / \mathfrak{z}(\mathfrak{g})$ in place of $\mathfrak{f}$ and shows that there are two closed ideals $\mathfrak{j}$ and $\mathfrak{h}$ of $\mathfrak{g}$, both containing $\mathfrak{z}(\mathfrak{g})$ such that

$$
\mathfrak{g}=\mathfrak{i}+\mathfrak{h}, \quad \mathfrak{i} \cap \mathfrak{h}=\mathfrak{z}(\mathfrak{g}), \quad \mathfrak{n}(\mathfrak{g}) \subseteq \mathfrak{h}, \quad \text { and } \quad \operatorname{dim} \mathfrak{h} / \mathfrak{z}(\mathfrak{h})<\infty
$$

We notice that $[\mathfrak{j}, \mathfrak{h}] \subseteq \mathfrak{i} \cap \mathfrak{h}=\mathfrak{z}(\mathfrak{g})$. Thus the commutator function $(x, y) \mapsto[x, y]$ : $\mathfrak{i} \times \mathfrak{h} \rightarrow \mathfrak{z}(\mathfrak{g})$ is a $\mathbb{Z}$-bilinear continuous morphism. Thus for each $b \in \mathfrak{h}$ the morphism $x \mapsto[x, b]: \mathrm{i} \rightarrow \mathfrak{z}(\mathfrak{g})$ vanishes on $\overline{[\mathrm{i}, \mathrm{i}]}$, and for each $a \in \mathfrak{j}$, the morphism $y \mapsto[a, y]$ : $\mathfrak{h} \rightarrow \mathfrak{z}(\mathfrak{g})$ vanishes on $\overline{[\mathfrak{h}, \mathfrak{h}]}$. Thus

$$
[\overline{[\mathfrak{i}, \dot{\mathfrak{i}}}], \mathfrak{h}]+[\dot{\mathfrak{i}},[\mathfrak{h}, \mathfrak{h}]]=\{0\} .
$$

Next we discuss the ideal $\mathrm{j}$ : The nilradical $\mathrm{n}(\mathrm{i})$ of $\mathrm{i}$ is a characteristic ideal and is therefore a pronilpotent ideal of $\mathfrak{g}$. Hence it is contained in $\mathfrak{n}(\mathfrak{g}) \subseteq \mathfrak{h}$, and so it is contained in fact in $\mathfrak{i} \cap \mathfrak{h}=\mathfrak{z}(\mathfrak{g})$. Since $\mathfrak{n}_{\text {cored }}(\mathfrak{i}) \subseteq \mathfrak{n}(\mathfrak{i})$ this entails $\mathfrak{n}_{\text {cored }}(\mathfrak{i}) \subseteq \mathfrak{z}(\mathfrak{i})$. Now Lemma 2.10 applies to $j$ and shows that $r(i)$ is nilpotent of class $\leq 2$ and

$$
\mathrm{i}=\mathfrak{r}(\mathrm{i}) \oplus \mathfrak{s}(\mathrm{i}),
$$

a direct sum with a unique Levi summand $\mathfrak{s}(\mathfrak{i})$. Now $\mathfrak{s}(\mathfrak{i}) \subseteq \overline{[\mathfrak{i}, \hat{i}]}$ commutes elementwise with $\mathfrak{h}$ by $(2)$ and with $\mathfrak{r}(\mathfrak{i})$ by $(3)$. Hence it is an ideal of $\mathfrak{g}=\mathfrak{i}+\mathfrak{h}$ while $\mathfrak{g}$ is the semidirect sum of $\mathfrak{r}(\mathfrak{g})$ and any Levi summand $\mathfrak{s}$ by the Levi-Mal'cev Theorem 7.52(i) of [9]. Now $\mathfrak{s}(\mathrm{i}) \subseteq \mathfrak{s}$ by the Levi-Mal'cev Theorem 7.77(iv) of [9]. Since $\mathfrak{s}$ is semisimple, $\mathfrak{s}$ is a direct sum $\mathfrak{s}(\mathrm{i}) \oplus \mathrm{t}$ with a semisimple direct factor $\mathrm{t}$ of $\mathfrak{s}$, and so $\mathfrak{g}=\mathfrak{s}(\mathfrak{i}) \oplus \mathfrak{g}_{1}$ where the ideal direct summand $\mathfrak{g}_{1}$ is $\mathfrak{r}(\mathfrak{g}) \oplus \mathfrak{t}$. If $\mathfrak{g}_{1}$ has the structure asserted in Statement (ii) of the Theorem, then (ii) holds for $\mathfrak{g}$. We may and will therefore assume that $\mathfrak{s}(\mathrm{i})=\{0\}$, that is, that $\mathrm{i}$ is nilpotent of class $\leq 2$, that is, $\mathfrak{i}=\mathfrak{n}(\mathfrak{i})$ and $\operatorname{dim} \mathfrak{j} / \mathfrak{z}(\mathfrak{g})<\infty$. Then $(1)$ implies $\operatorname{dim} \mathfrak{g} / \mathfrak{\jmath}(\mathfrak{g})<\infty$.

By Proposition 2.7 applied to $\mathfrak{g}$ where $\mathfrak{z}(\mathfrak{g})$ takes the place of $\mathfrak{z}$, there is a finitedimensional ideal $\mathfrak{f}$ of $\mathfrak{g}$ containing $\mathfrak{g}^{\prime}=\mathfrak{s}+\mathfrak{n}_{\text {cored }}(\mathfrak{g}$ ) (where $\mathfrak{s}$ is any Levi summand of $\mathfrak{g})$, and there is a central ideal $\mathfrak{a} \subseteq \mathfrak{z}(\mathfrak{g})$ such that

$$
\mathfrak{g}=\mathfrak{a} \oplus \mathfrak{\mathfrak { T }}=(\mathfrak{a} \oplus \mathfrak{r}(\mathfrak{\mathfrak { T }})) \oplus \mathfrak{s} .
$$

This completes the proof of (ii).

In Example 2.5(iii) we saw that for every infinite-dimensional weakly complete topological vector space $V$, the class 2 nilpotent pro-Heisenberg algebra $\mathfrak{h}(V)$ fails 
to be very rich, and its nilpotent core nilcore $(\mathfrak{h}(V))$ is isomorphic to $V$. This example shows that Theorem 2.11 is likely to come very close to the best possible if one looks for a reasonably general sufficient condition for a pro-Lie algebra to be very rich.

\section{Splitting on the Group Level}

We now return from the pure algebra level to the pro-Lie group level. Recall that the nilradical $N(G)$ of a connected pro-Lie group is the largest connected pronilpotent normal subgroup. We notice that if $G$ is a pro-Lie group, then $N\left(G_{0}\right) / Z\left(G_{0}\right)_{0}$ is a pro-Lie group by Theorem 4.28(i) of [9], where it is shown that the quotient of a connected pro-Lie group modulo a connected closed normal subgroup is a pro-Lie group. This pro-Lie group is called the nilcore of $G$. It is written nilcore $(G)$.

Proposition 3.1. Let $G$ be a connected pro-Lie group. Then

(i) its nilcore nilcore $(G)$ is a simply connected pronilpotent pro-Lie group.

(ii) Its Lie algebra $\mathfrak{L}(\operatorname{nilcore}(G))$ is naturally isomorphic to the nilcore nilcore $(\mathfrak{g})$ of its Lie algebra $\mathfrak{g}=\mathfrak{Q}(G)$.

(iii) The function exp : (nilcore $(\mathfrak{g}), *) \rightarrow \operatorname{nilcore}(G)$ is a natural isomorphism of proLie groups, where $*$ denotes the Campbell-Hausdorff multiplication on the pro-Lie algebra nilcore $(\mathfrak{g})$.

Proof. By Theorem 9.50 of [9], the maximal compact subgroup of $N(G)$ is contained in the center $Z(G)$. From 11.26 of [9] it follows that nilcore $(G)$ has no nontrivial compact subgroups. Then (i) and (iii) follow from Theorem 11.27 of [9].

(ii) By Theorem 10.42 of [9] we have $\mathfrak{L}(N(G))=\mathfrak{n}(\mathfrak{g})$, and by Proposition 9.23 we know $\mathfrak{L}(Z(G))=\mathfrak{z}(\mathfrak{g})$. Now [4], Corollary 6.7(i) or Corollary 4.21(i) proves the claim.

(iii) A combination of Theorem 8.5, Proposition 8.8, Theorem 8.15 of [9] shows, that for a simply connected nilpotent pro-Lie Group $N$, the exponential function $\exp _{N}:(\mathfrak{L}(N), *) \rightarrow N$ is an isomorphism of topological groups. The assertion is now a consequence of conclusion (iii) above.

The structure of the nilcore of a connected pro-Lie group is therefore completely known when its Lie algebra is known. Trivially, $Z(G)_{0} \subseteq N(G) \cap Z(G)$. Conversely, $N(G) \cap Z(G) \subseteq Z(N(G))$, and by Proposition 11.20(i) of [9], the center of $N(G)$ is connected. So

$$
Z(G)_{0} \subseteq N(G) \cap Z(G) \subseteq Z(N)_{0},
$$

but Example 2.5(ii) shows that $Z(G)=\{0\}$ and $Z(N)_{0}=Z(N) \neq\{0\}$ may occur. Since $N(G) / Z(N(G))$ is a quotient of nilcore $(G)$, the finite-dimensionality of $N(G) / Z(N(G))$ is a weaker condition than that of nilcore $(G)$. 
We are now ready for our general pro-Lie group version of the Iwasawa Local Splitting Theorem.

Theorem 3.2 (Local Splitting Theorem). Let G be a pro-Lie group whose nilcore

$$
\operatorname{nilcore}(G) \stackrel{\text { def }}{=} N\left(G_{0}\right) / Z\left(G_{0}\right)_{0}
$$

is finite-dimensional. Then every identity neighborhood contains a closed normal almost connected subgroup $N$ such that $G / N$ is a Lie group and that there is a morphism $\varphi: \widehat{G / N} \rightarrow G$ such that the morphism $\mu: N \times \widehat{G / N} \rightarrow G, \mu(n, x)=n \varphi(x)$ is an open morphism having a discrete kernel. In particular, $G, N \times \widetilde{G / N}$, and $N \times G / N$ are locally isomorphic.

Proof. Let $\mathfrak{g} \stackrel{\text { def }}{=} \mathfrak{Q}(G)$ be the Lie algebra of $G$. By Proposition 3.1 , nilcore $(\mathfrak{g})=\mathfrak{g} / \mathfrak{n}(\mathfrak{g})$ is finite-dimensional. Then by 2.11, the Structure Theorem of Pro-Lie algebras with Finite-Dimensional Nilcore, $\mathfrak{g}$ is extremely rich (see Definition 2.4.) Thus all sufficiently small cofinite-dimensional ideals are well-complemented. By [4], Corollary 6.7(ii) or by Corollary 4.21(ii) of [9], $\{\mathfrak{Q}(N) \mid N \in \mathscr{N}(G)\}$ converges to zero. Hence for all sufficiently small $N \in \mathscr{N}(G)$, the ideal $\mathfrak{I}(N)$ is well-complemented. Now Theorem 1.3 of the Local Splitting of Lie Group Quotients and its Corollary 1.4 together prove the Theorem.

The following discussion requires the theory of analytic subgroups of pro-Lie groups which we expounded in Chapter 9 of [9]. By the definition of analytic subgroups in [9] (see Definition 9.5, Proposition 9.6), $\operatorname{im}(\varphi)$ is an analytic subgroup whose Lie algebra $\mathfrak{f}$ is the direct summand for which $\mathfrak{g}=\mathfrak{n} \oplus \mathfrak{f}$. We note that $(n, x) \in \operatorname{ker} \mu$ iff $n=\varphi(x)^{-1} \in$ $N \cap \operatorname{im} \varphi=A(\mathfrak{f})$. That is, $x \mapsto\left(\varphi(x)^{-1}, x\right): \varphi^{-1}(N) \rightarrow \operatorname{ker} \mu$ is an isomorphism. Let $L$ be the underlying Lie group of the analytic group $A(\mathfrak{f})$. Then, the morphism $(n, \ell) \mapsto$ $n \ell: N \times L \rightarrow G$ is a surjective morphism implementing a local isomorphism. Its kernel $\left\{\left(\ell^{-1}, \ell\right) \mid \ell \in N \cap A(\mathfrak{f})\right\}$ is algebraically isomorphic to a discrete and hence central normal subgroup of $L$ and is therefore a finitely generated abelian group; it is algebraically isomorphic to $N \cap A(\mathfrak{f})$.

We retain the notation of the Local Splitting Theorem 3.2 and provide additional information on the local splitting.

Proposition 3.3 (Sandwich Theorem for Local Splitting). Let G be a connected pro-Lie group whose nilcore nilcore $(G) \stackrel{\text { def }}{=} N(G) / Z(G)_{0}$ is finite-dimensional. We assume that $A(\mathfrak{f})=\operatorname{im} \varphi$ is a closed analytic subgroup L. Then 3.2 applies, and the quotient map $q: G \rightarrow G / D, D=N \cap L$ implements a covering morphism. Further,

$$
v: N / D \times L / D \rightarrow G / D, \quad v((n D, \ell D))=n \ell D,
$$

is an isomorphism. Let $q_{N}: N \rightarrow N / D$ and $\pi_{L / D}: \widetilde{G / N} \rightarrow L / D$ be the quotient morphisms, each with kernel isomorphic to $D$. Then the sandwich diagram 


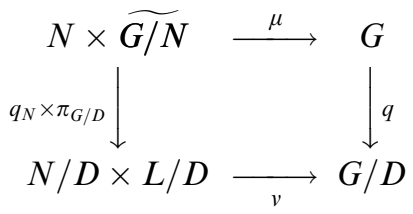

commutes.

Proof. Given the information in Theorem 3.2, all of the assertions of the corollary amount to a straightforward verification.

We should record, that the Structure Theorem of Pro-Lie algebras with FiniteDimensional Nilcore 2.11 contains some information which is not yet reflected in the theorems that we have formulated so far.

Corollary 3.4 (The Structure Theorem for Groups with Finite-Dimensional Nilcore). Let $G$ be a connected pro-Lie group. Then the following statements are equivalent:

(i) The nilcore nilcore $(G)$ is finite-dimensional.

(ii) $G$ is locally isomorphic to the product of a closed normal almost connected subgroup $N$, whose identity component $N_{0}$ is reductive, and a connected Lie subgroup $L$.

Proof. (i) $\Rightarrow$ (ii): By the Local Splitting Theorem 3.2 we find a closed normal subgroup $N$ (which we may choose as small as we like) a connected Lie group $L$ such that $N \times L$ and $G$ are locally isomorphic and that $\mathfrak{g}=\mathfrak{n} \oplus \mathfrak{f}$ where $\mathfrak{n}=\mathfrak{L}(N)$ and $\mathfrak{f}=\mathfrak{L}(L)$. By Theorem 2.11, if $N$ and then $n$ is small enough, $\mathfrak{n}$ is an ideal of a reductive algebra and is, therefore, reductive itself. By Theorem 10.29(i) of [9], this shows that $N_{0}$ is reductive.

(ii) $\Rightarrow$ (i): If (ii) holds, then $\mathfrak{g}=\mathfrak{n} \oplus \mathfrak{\mathfrak { f }}$ with a reductive Lie algebra $\mathfrak{n}=\mathfrak{Q}(N)$ and a finite-dimensional ideal $\mathfrak{f}=\mathfrak{L}(L)$. Then nilcore $(\mathfrak{g})=\operatorname{nilcore}(\mathfrak{n}) \oplus \operatorname{nilcore}(\mathfrak{f})=$ nilcore $(f)$, is finite-dimensional since $\mathfrak{f}$ is finite-dimensional.

Information on the structure of reductive connected pro-Lie groups is to be found in [9] in Theorems 10.29, 10.32, 10.48, and 11.8.

Our discussion now leads back to the domain of locally compact groups, where the original theorem of Iwasawa's started. It was concerned with connected locally compact groups. The following consequence of our present theory is, therefore, more general.

Corollary 3.5 (Strong Iwasawa Local Splitting Theorem). Let $G$ be a locally compact pro-Lie group. Then there is an open subgroup $\mathbb{G}$ of $G$ and there are arbitrarily small compact normal subgroups $N$ of $\mathbb{G}$ such that $\mathbb{G} / N$ is a Lie group and $G$ and $N \times \mathbb{G} / N$ are locally isomorphic. 
Proof. We recall from [11], p. 175, that a locally compact group is automatically a pro-Lie group if it is almost connected. So let $\mathbb{G}$ be an open almost connected subgroup of $G$ which always exists because every locally compact totally disconnected group (such as for instance $G / G_{0}$ ) has compact open subgroups.

Let $\mathfrak{g}=\mathfrak{L}(G)=\mathfrak{L}(\mathbb{G})$. By Corollary 12.88 of [9], $\mathfrak{g} / \mathfrak{m}(\mathfrak{g})$ the factor algebra modulo the largest compactly embedded ideal is finite-dimensional. Hence from the Structure of Pro-Lie Algebras with Finite-Dimensional Nilcore 2.11 and from Proposition 3.1(ii), we know that nilcore $(\mathfrak{g})=\mathfrak{L}($ nilcore $(G))$ is finite-dimensional. So the Local Splitting Theorem 3.2 applies to $\mathbb{G}$ and proves the assertion.

\section{Additional Structural Information}

The hypotheses of the Local Splitting Theorem 3.2 allow us to draw additional structural conclusions which are important.

In Chapter 12 of [9] we have discussed the special structure of centrally supplemented groups: see [9], Definition 12.72ff. We recall that every connected pro-Lie group $G$ has a unique characteristic largest compact normal abelian and hence central subgroup $\mathrm{KZ}(G)$, and that $G / \mathrm{KZ}(G)$ has no nontrivial compact central subgroups (see [9], Definition 9.47ff., notably Theorem 9.50). Recall from Theorem 3.1 that we denote by $A(\mathfrak{g})$ the unique smallest analytic subgroup of $G$ having $\mathfrak{g}$ as its Lie algebra.

Theorem 4.1 (Theorem on Central Supplementation). Let G be a connected pro-Lie group whose nilcore nilcore $(G) \stackrel{\text { def }}{=} N(G) / Z(G)_{0}$ is finite-dimensional. Then

$$
G=\mathrm{KZ}(G) A(\mathfrak{g})
$$

In particular, $G$ is centrally supplemented.

Proof. We factor $\mathrm{KZ}(G)$ and assume $\mathrm{KZ}(Z)=\{1\}$; we must show $G=A(\mathfrak{g})$. Let $\mathfrak{g}=\mathfrak{j} \oplus \mathfrak{h}$ where $\mathfrak{j}$ is a reductive ideal and $\mathfrak{h}$ is a finite-dimensional ideal according to Theorem 2.10. Since $\{\mathfrak{L}(N) \mid N \in \mathscr{N}(G)\}$ converges to 0 in $\mathfrak{g}$ by [4], Corollary 6.7(ii) or [9], 4.21(ii), there is an $N \in \mathscr{N}(G)$ such that $\mathfrak{L}(N) \subseteq \mathrm{i}$. Since i is a product of simple Lie algebras and copies of $\mathbb{R}$ by the Structure Theorem of Reductive Pro-Lie algebras 7.27 in [9], we have $\mathfrak{j}=\mathfrak{L}(N) \oplus \mathfrak{f}$ with a finite-dimensional semisimple ideal $\mathfrak{f}$ and so we have $\mathfrak{g}=\mathfrak{L}(N) \oplus \mathfrak{f} \oplus \mathfrak{h}$. It is therefore no loss of generality if we assume henceforth that there is an $N \in \mathscr{N}(G)$ such that $\mathfrak{L}(N)=\mathrm{j}$. Then $G / N$ is a Lie group and thus

$$
G=N A(\mathfrak{g})
$$

by [4] Corollary 6.8(iii) or by Corollary 4.22(iii) of [9].

The analytic subgroup $A(\mathfrak{g})$ is the product of the normal analytic subgroups $A(\mathfrak{i})$ and $A(\mathfrak{h})$ which commute elementwise. By [4], 6.8(i) or by [9], 4.22(i), $N_{0}=\overline{A(\mathrm{i})}$. Then $A(\mathfrak{h}) \subseteq Z\left(N_{0}, G\right)$ and thus $G=N_{0} Z\left(N_{0}, G\right)$ and $Z\left(N_{0}\right) \subseteq Z(G)$. Further, $N_{0}$ 
is reductive by Theorem 10.48 of [9], and therefore satisfies $N_{0}=\mathrm{KZ}\left(N_{0}\right) A\left(\mathrm{i}, N_{0}\right)$ and is centrally supplemented in its own right. Since $\mathrm{KZ}\left(N_{0}\right) \subseteq \mathrm{KZ}(G)=\{1\}$, we have

$$
N_{0}=A(\mathrm{i}) \subseteq A(\mathrm{i}) A(\mathfrak{h})=A(\mathfrak{g}) .
$$

Then (1) implies

$$
G=N A(\mathfrak{h}) .
$$

Now $\mathfrak{L}\left(G / N_{0}\right)=\mathfrak{L}(G) / \mathfrak{L}\left(N_{0}\right)=\mathfrak{L}(G) / \mathfrak{L}(N)=\mathfrak{g} / \mathfrak{i} \cong \mathfrak{h}$ by Corollary 6.7(i) of [4] or Corollary 4.21(i) of [9]. Therefore $G / N_{0}$ is finite-dimensional by Definition (see [9], 9.43). Then $G / N_{0}$ is locally compact metric by [9], Theorem 9.44 , and we may assume that $N / N_{0}$ is compact totally disconnected. Let $\varphi: G / N=\Gamma(\mathfrak{h}) \rightarrow G$ be a morphism such that $\operatorname{im} \varphi=A(\mathfrak{h})$. Then $Z \stackrel{\text { def }}{=} \varphi^{-1}(N)$ is a discrete central subgroup $Z$ of $\widetilde{G / N}$ and is therefore finitely generated. Thus Theorem 5.32(iv) of [9] applies to $A \stackrel{\text { def }}{=} \overline{\varphi(Z)}$ and shows that $A \cong \operatorname{comp}(A) \times \mathbb{Z}^{n}$ for some nonnegative integer. However, $A \subseteq Z(G)$ whence $\operatorname{comp}(A) \subseteq \mathrm{KZ}(G)=\{1\}$. Thus $A$ is free discrete and therefore agrees with $\varphi(Z)=N \cap A(\mathfrak{h})$. Then the map $i: A(\mathfrak{h}) / A \rightarrow G / N, i(a A)=a N$ is a bijective morphism by (3). Let $\psi$ be the corestriction of $\varphi$ to its image. Then the composition

$$
\widetilde{G / N} \stackrel{\psi}{\longrightarrow} A(\mathfrak{h}) \stackrel{\text { quot }}{\longrightarrow} A(\mathfrak{h}) / A \stackrel{i}{\longrightarrow} G / N
$$

is a surjective morphism between locally isomorphic Lie groups and is, therefore, open and implements itself a local isomorphism. This implies that $\psi$ is open and $H \stackrel{\text { def }}{=} A(\mathfrak{h})$ is a Lie group and $G=N H, N \cap H=A$. Thus $N / A \cong G / H$ is connected and is locally isomorphic to $N$ and is, therefore a connected pro-Lie group. By (2) $N_{0}=\left\langle\exp _{N} \mathrm{i}\right\rangle$ and by [4] Corollary 6.8(iii) or by Corollary 4.22(iii) of [9], $\left\langle\operatorname{im} \exp _{N / A}\right\rangle=N_{0} A$. Therefore $N_{0} A / N_{0}$ is a connected space which is completely regular as a Hausdorff topological group. Its cardinality is therefore 1 or at least $2^{\aleph_{0}}$. Since $A$ is countable, it follows that $N \cap H=A \subseteq N_{0}$. By Theorem 9.44 of [9], $A N_{0} / N_{0}=\varphi(Z) N_{0} / N_{0}$ is dense in $N / N_{0}$ and therefore $N=N_{0}$, that is $N=A(\mathrm{i})$. Now (1) shows that $G=A(\mathfrak{g})$ which is what we had to show.

From [2], Theorem 8.20(i) it follows that every compact abelian group $K$ contains a compact totally disconnected subgroup $D$ such that $K=K_{a} D$ (see also [2], Theorem 9.41). Therefore, in the circumstances of Theorem 4.1, we find a compact zerodimensional central subgroup $D$ of $G$ such that $G=D A(\mathfrak{g})=D\left\langle\exp _{G} \mathfrak{g}\right\rangle$. We can therefore write

Corollary 4.2. Let $G$ be a pro-Lie group with a finite-dimensional nilcore. Then there is a compact totally disconnected central subgroup $D$ of $G$ and an open surjective morphism 


$$
\mu: D \times \tilde{G} \rightarrow G, \quad \mu(d, x)=d \pi_{G}(x)
$$

with a prodiscrete kernel.

Proof. We recall that we have a universal morphism $\pi_{G}: \tilde{G} \rightarrow G$ whose image is exactly $A(\mathfrak{g})=\left\langle\operatorname{im}_{\exp }\right\rangle$. The existence of the surjective morphism $\mu$ then follows from the preceding remarks in view of Theorem 4.1. The Open Mapping Theorem 9.60 of [9] finally shows that $\mu$ is open.

Since every connected locally compact group is a pro-Lie group by Yamabe's Theorem, and since the nilcore of a locally compact group is always finite dimensional, Corollary 4.2 implies at once the following consequence:

Corollary 4.3. Let $G$ be a connected locally compact group. Then there is a compact totally disconnected central subgroup $D$ of $G$ and an open surjective morphism

$$
\mu: D \times \tilde{G} \rightarrow G, \quad \mu(d, x)=d \pi_{G}(x)
$$

with a prodiscrete kernel.

We should recall that $\tilde{G}$, while being a simply connected pro-Lie group, is in general not locally compact. This is illustrated by any infinite dimensional compact connected abelian group $G$, for which $\tilde{G}$ is the additive group of the pro-Lie algebra $\mathfrak{L}(G) \cong \mathbb{R}^{J}, \operatorname{card} J=\operatorname{dim} G$. (See [2], Chapter 8, notably 8.20.)

\section{Some Comments on Connectedness}

We observe that, classically, the Iwasawa Local Splitting Theorem 3.5 required the hypothesis of connectivity. The presentation of the local splitting theory in the framework of pro-Lie group theory, culminating in Theorem 3.2 does not require this hypothesis, as long as we are dealing with pro-Lie groups. This is plausible because the conclusion is a local one. The global version concludes the existence of an open morphism $N \times G / N \rightarrow G$, and not a surjective one. We should recall that by the definition of the universal group $\tilde{H}$ of a pro-Lie group $H$ (see [9], Theorem 6.6ff.) it is always a connected, indeed simply connected group regardless of whether $H$ is connected or not. We distinguish "universal group" of $H$ from "universal covering group" of $H$. Indeed if the underlying space of $H$ has a universal covering space, then the two concepts agree (see Theorem 8.21 of [9]). In particular, if $H$ is a Lie group (such as in the case for $G / N$ whenever $N \in \mathscr{N}(G)$ ), then $\tilde{H}$ is the universal covering group of $H_{0}$.

In the case of a locally compact group $G$, by the results of Yamabe, there is always an open subgroup $\mathbb{G}$ of $G$ which is a pro-Lie group. Then the pro-Lie group result applies to $\mathbb{G}$ and produces arbitrarily small normal subgroups $N$ of $\mathbb{G}$ such that $\mathbb{G}$ and $N \times \mathbb{G} / N$ are locally isomorphic. But since $G$ and $\mathbb{G}$ trivially are locally isomorphic, 
one can still say that a locally compact group, quite generally, is locally isomorphic to the product of an arbitrarily small compact subgroup (not necessarily normal!) and some Lie group.

In [3] this was argued directly from the "connected" version of Iwasawa's Local Splitting Theorem for Locally Compact Groups; but that required some technical ad hoc arguments.

\section{References}

[1] George Michael A. A.: On Inverse Limits of Finite Dimensional Lie Groups. J. Lie Theory 16 (2006), 221-224

[2] Hofmann K. H., and Morris S. A.: The Structure of Compact Groups. Walter de Gruyter, Berlin $2006^{2}$, xviii+858pp.

[3] — : Transitive Actions of Compact Groups and Topological Dimension. J. Algebra 234 (2000), 454-479

[4] - Projective Limits of Finite Dimensional Lie Groups. Proc. London Math. Soc. (3) 87 (2003), 647-676

[5] —: The Structure of Abelian Pro-Lie Groups. Math. Z. 248 (2004), 867-891

[6] - Sophus Lie's Third Fundamental Theorem and the Adjoint Functor Theorem. J. Group Theory 8 (2005), 115-133

[7] —: An Open Mapping Theorem for Pro-Lie Groups. J. Austral. Math. Soc.; to appear

[8] — : An Open Mapping Theorem for Topological Groups; submitted

[9] —: The Lie Theory of Connected Pro-Lie Groups - A Structure Theory for Pro-Lie Algebras, Pro-Lie Groups and Connected Locally Compact Groups. EMS Publishing House, Zürich 2007, xvi+675pp.; to appear

[10] Iwasawa K.: On some types of topological groups. Ann. of Math. 50 (1949), 507-558

[11] Montgomery D., and Zippin L.: Topological Transformation Groups. Interscience Publishers, New York 1955

[12] Roelke W., and Dierolf S.: Uniform Structures on Topological Groups and their Quotients. McGraw-Hill, New York etc. 1981

[13] Yamabe H.: On the Conjecture of Iwasawa and Gleason. Ann. of Math. 58 (1953), 48-54

[14] - Generalization of a theorem of Gleason. Ann. of Math. 58 (1953), 351-365

Received 5 December 2005; in final form 16 November 2006

Karl H. Hofmann, Fachbereich Mathematik, Darmstadt University of Technology, Schlossgartenstr. 7, D-64289 Darmstadt, Germany

hofmann@mathematik.tu-darmstadt.de

Sidney A. Morris, School of Information Technology and Mathematical Sciences, University of Ballarat, P.O. Box 663, Ballarat, Victoria 3353, Australia

s.morris@ballarat.edu.au 
Copyright of Forum Mathematicum is the property of Walter de Gruyter GmbH \& Co. KG. and its content may not be copied or emailed to multiple sites or posted to a listserv without the copyright holder's express written permission. However, users may print, download, or email articles for individual use. 